Appeared in Communications in Pure and Applied Mathematics volume 51, pages 733-795, 1998.

\title{
SINGULARITY FORMATION IN THIN JETS WITH SURFACE TENSION
}

\author{
M. C. PUGH AND M. J. SHELLEY
}

\begin{abstract}
We derive and study asymptotic models for the dynamics of a thin jet of fluid that is separated from an outer immiscible fluid by fluid interfaces with surface tension. Both fluids are assumed to be incompressible, inviscid, irrotational, and density matched. One such thin jet model is a coupled system of PDEs with nonlocal terms - Hilbert transforms - that result from expansion of a Biot-Savart integral. In order to make the asymptotic model well-posed, the Hilbert transforms act upon time derivatives of the jet thickness, making the system implicit. Within this thin jet model, we demonstrate numerically the formation of finite-time pinching singularities, where the width of the jet collapses to zero at a point. These singularities are driven by the surface tension, and are very similar to those observed previously by Hou, Lowengrub, and Shelley in large-scale simulations of the Kelvin-Helmholtz instability with surface tension, and in other related studies. Dropping the nonlocal terms of the model, we also study a much simpler local model. For this local model we can preclude analytically the formation of certain types of singularities, though not those of pinching type. Surprisingly, we find that this local model forms pinching singularities of a very similar type to those of the nonlocal thin jet model.
\end{abstract}

\section{INTRODUCTION}

A class of fluid phenomena for which hydrodynamic singularities play a demonstrably central role is the reconfiguration of fluid masses that are bounded or separated by an interface under surface tension. The pinching-off of a droplet from a stream is the most common of examples. In continuum models such as the Navier-Stokes or Euler equations, the collapse of the distance between the bounding interfaces implies (at the very least) a pointwise divergence of fluid velocity gradients. This is worth understanding since both the collapse and the divergence of velocity gradients point to possible limitations of continuum modeling, since arbitrarily small length-scales become important and neglected molecular processes must come into play. Furthermore, such collisions and ensuing reconnections are the mediating events through which a flow reorganizes its global structure. The phenomena of vortex reconnection in nearly inviscid fluids, and its possible relation to vorticity blow-up in Euler fluids, has a similarly intriguing aspect.

Singularities occurring through the collision of bounding interfaces under surface tension are especially intriguing since surface tension is often viewed as a regularizing force - the force that takes nearly flat interfaces and makes them flatter. However, it is often the case that surface tension itself drives (or at least mediates) the collision - the Rayleigh instability of an axisymmetric stream is a classical example. Also, depending on the assumed physics of the flow, surface tension can enter the dynamics in varied ways, e.g. dissipatively or dispersively. Here, we consider a situation where surface tension enters dispersively as a linearly regularizing effect, but nonetheless strongly drives the collision process.

Our motivating example comes from numerical studies by Hou, Lowengrub, \& Shelley $([20,21]$, referred to as HLS1 and HLS2, respectively) of the effect of surface tension on the Kelvin-Helmholtz instability of an interface between two immiscible, two-dimensional, irrotational, inviscid, density-matched fluids. In this context, the interface is a vortex sheet [36]. In the absence of surface tension, the interface is linearly unstable at all length scales, and the problem is ill-posed in the sense of Hadamard. Analytical and numerical studies $[31,30,25,37]$ have shown that at times well before the development of any large-scale structure, a rapid concentration of circulation along the interface leads generically to the formation of an isolated curvature singularity in finite time. (Nonetheless, Delort has shown that a vortex sheet that is single signed in its circulation will exist globally in time as a weak solution to the Euler equations [13].) Various regularizations

Date: December 20, 1997. 
- smoothing the vorticity [3, 42], blob smoothing of the Biot-Savart integral [24], the inclusion of viscosity [42] - remove the catastrophic instability and the singularity. The "interface" is then observed to roll-up and form the Kelvin-Helmholtz spirals associated typically with a shear layer.

Surface tension regularizes the Kelvin-Helmholtz instability by introducing dispersion at small length scales $[2,4,20]$. In HLS2 [21] the authors give numerical evidence for the removal of the Kelvin-Helmholtz singularity, and observe the ensuing roll-up of the interface. However, they also find that at sufficiently large Weber numbers, the presence of surface tension will produce oppositely signed circulation from initially single signed circulation. This then couples together adjacent turns of the spiral and generates strong localized fluid jets. These jets amplify and collapse in width until the interface self-intersects, forming trapped bubbles of fluid at the core of the spiral. The HLS2 results suggest that at the time of the collision, the interface forms corners with the fluid velocity diverging simultaneously. We call such singularities, which signal a possible change of topology, "pinching singularities". Figure 1 shows such a simulation from HLS2. If the collapse were of a

(a)

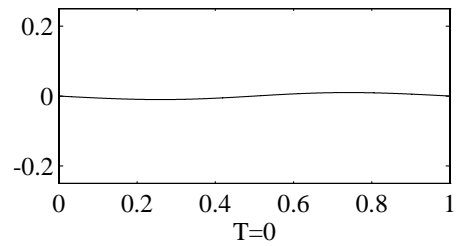

(c)

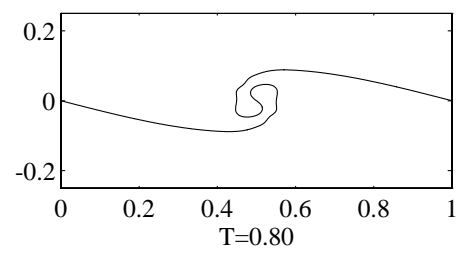

(e)

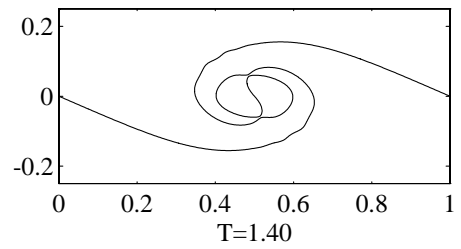

(b)

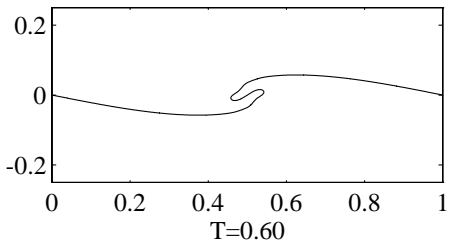

(d)

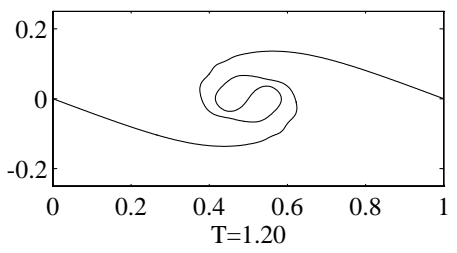

(f)

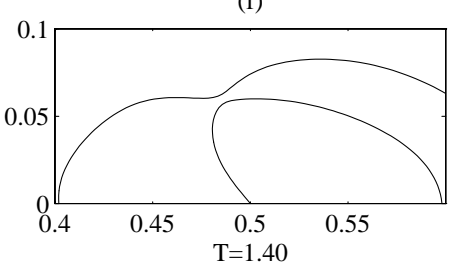

Figure 1. The long-time evolution of an initially nearly flat vortex sheet for $W e=200$. The bottom-right box (f) shows a close-up of the thinning neck at $t=1.4$ [21].

self-similar form, then one would expect that

$$
d_{\min } \sim\left(t_{p}-t\right)^{2 / 3}, \gamma_{\max } \sim\left(t_{p}-t\right)^{-1 / 3} \text { and } \kappa_{\max } \sim\left(t_{p}-t\right)^{-2 / 3}
$$

where $d_{\min }$ is the width of the fluid neck, $\gamma$ is the vortex sheet strength, and $\kappa$ the interfacial curvature. (See Keller \& Miksis [23] for an analysis of the related problem of the fissioning of two fluid masses touching initially at corners.) HLS2 find that $d_{m i n}$ shows close conformance to this prediction of self-similarity, but also that $\gamma$ and $\kappa$ show persistent discrepancies. These differences could result from the presence of higher-order contributions not accounted for in their fitting procedures, or from not being able to compute sufficiently close to the singularity time with good numerical resolution. Figure 2 shows two simulations of a periodic symmetric jet with surface tension and also shows self-intersection [21, 27]. Numerical studies of the Rayleigh-Taylor instability $[20,19]$ in the presence of surface tension also show the formation of pinching singularities very similar to those seen in HLS2. 

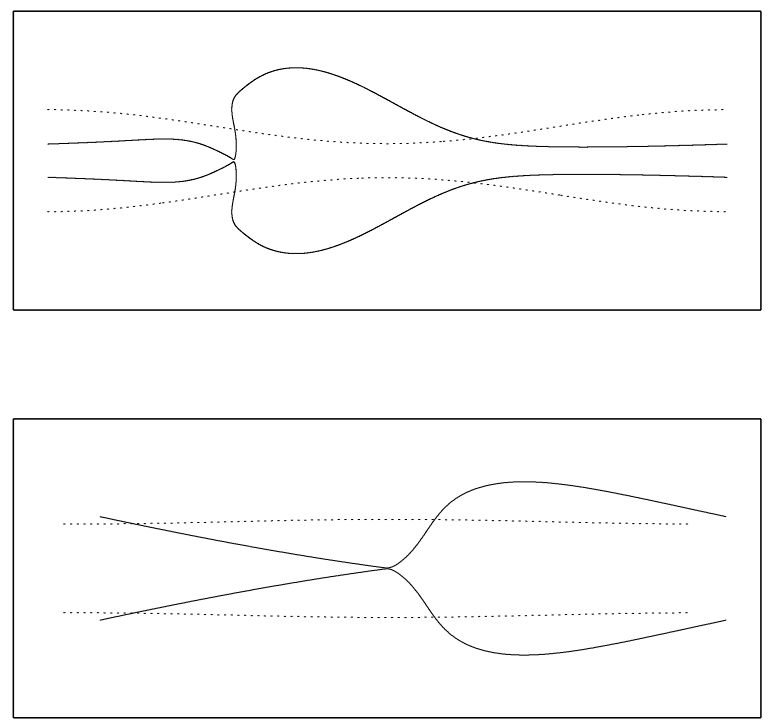

Figure 2. Top: The formation of a pinching singularity in a symmetric jet bounded by two vortex sheets with surface tension. The Weber number is chosen so there are many unstable modes, causing the "slop-over" near the pinching region [27]. Bottom: Here, the Weber number has been chosen so there are few unstable modes [21].

In this paper, we abstract what seems to be the crucial ingredient of the pinching singularity observed by HLS2, and study the role of surface tension on the dynamics of an isolated jet between two vortex sheets with surface tension. In this setting, the vortex sheets separate an inner fluid from an outer immiscible fluid. As in HLS2, we consider the two fluids to be density matched since density stratification does not apparently modify the form of the singularity [19], and also to make the physical situation as reduced as possible. As a further simplification, we study the dynamics of thin symmetric jets, using a large aspect ratio expansion to derive reduced PDE descriptions for the jet width $h(x, t)$ and sheet strength $\gamma(x, t)$. We derive one reduced system that captures the competition between the Kelvin-Helmholtz instability of a jet, and the dispersive effect of surface tension. Moreover, our numerical simulations show that this system also forms corner pinching singularities in finite time, as in HLS1. But in contrast to HLS2, we find that $\gamma$ and $h_{x x}$ (the "long-wave" curvature) now behave roughly in accordance with self-similarity in their temporal behavior, while $h_{\min }$ shows a persistent discrepancy. Perhaps these differences arise from the assumption of symmetry, not found in the well-analyzed full simulations of HLS1, though we note that if the singularities of the reduced system are of a self-similar type, then the asymptotic assumptions made to derive the reduced system are violated. Of course, a violation of the asymptotic assumptions does not imply a priori that such models fail to capture the basic form of the singularity of the original system; We will address this point in a future study comparing our model equations with simulations of a symmetric, pinching jet in the unapproximated system [22].

In Section 2 we give a new formulation of the Euler equations for the dynamics of a symmetric jet bounded by fluid interfaces that separate two immiscible, density-matched, irrotational fluids. In Section 3 we show that this formulation admits a shallow-water expansion in a strikingly direct manner, but that the expansion yields a system which is ill-posed while the full system is not. The cause of this ill-posedness is clear and can be removed in several ways. One is by making the system implicit, yielding the thin jet model:

$$
\begin{aligned}
h_{t}+\left(h \mathcal{H}\left[h_{t}\right]\right)_{x} & =-(h \gamma)_{x} \\
\gamma_{t}+\left(\gamma \mathcal{H}\left[h_{t}\right]\right)_{x} & =-\gamma \gamma_{x}+W e^{-1} h_{x x x} .
\end{aligned}
$$

Here $2 h$ is the jet width, assumed to be initially positive, and $\gamma$ is the sheet strength, which is proportional to the velocity jump across the interface. The Weber number, $W e \propto 1 / \tau$, is a dimensionless quantity that 
measures the strength of inertial to surface tension forces. The Hilbert transform, $\mathcal{H}$, arises from the asymptotic expansion of the Biot-Savart integral [35]. This system fully determines exponents of self-similarity (1). These exponents are consistent with having a pinching singularity and agree with those for the unapproximated problem [21].

Equation (2) is a statement of mass conservation, and is in "shallow water form" $h_{t}+(h U)_{x}=0$. This form encodes the statement that the finite-time collapse of the jet width, $h \downarrow 0$, implies a flow singularity: a simple argument (see [12]) shows that if $h$ is smooth and $h(x, t) \downarrow 0$ at a point in finite time then, at the very least, $U_{x} \uparrow \infty$ at that point.

The thin jet model (2-3) captures the competition between the Kelvin-Helmholtz instability and the dispersive effect of surface tension. Numerical simulations show that the thin jet model produces corner pinching singularities, as observed in simulations of the full system (and, analogously to the full system, these pinching singularities disappear when surface tension is absent and a different non-pinching singularity occurs). However, careful data analysis of the spatial and temporal structure of the nascent singularity shows some discrepancies with the observations of HLS2, and with assumptions of self-similarity. Some of this may be associated with the flow straying from the shallow water regime. These results are presented in Section 5.

We also study whether the nonlocal terms of (2-3) are needed for a pinching singularity to occur. Retaining only the surface tension term from the higher-order asymptotic contributions, we have the local model, which we consider in Section 4:

$$
\begin{aligned}
h_{t}+\gamma h_{x} & =-h \gamma_{x} \\
\gamma_{t}+\gamma \gamma_{x} & =W e^{-1} h_{x x x}
\end{aligned}
$$

Here the surface tension contribution appears as a dispersive perturbation to a system that can be solved exactly in its absence. Specifically, for zero surface tension $(W e=\infty)$ exact solutions have finite-time singularities where $h \uparrow \infty$. We analytically preclude such finite-time blow-up for the local model in the presence of surface tension. We do prove that the above local model can have finite-time singularities in the presence of surface tension, but for a different class of initial data where both $h$ and $\gamma$ are compactly supported.

Simulations of this simpler system also show finite-time pinching singularities. Surprisingly, their structure is very similar to those of the thin jet model, even though this system apparently does not fully determine exponents of self-similarity. A possible mechanism for the singularity formation for this local model is discussed, though we have as yet no rigorous proof for its development. The numerical results for the local model are given in Subsection 5.4.

In Appendix A we derive a Fourier series reformulation of the boundary integral formulation for the motion of a symmetric jet and present its shallow water expansion. In Appendix B, we discuss a new data-fitting technique with which we fit the temporal behavior of extrema such as the minimum value of $h$ : $h_{\min }(t) \sim a\left(t_{c}-t\right)^{b}$. This technique gives us greater accuracy in determining exponents associated with the jet collapse. In Appendix $\mathrm{C}$ we discuss a similar fitting method for examining the spatial Fourier spectrum, which contains information about the spatial structure of the singularity.

1.1. Surface Tension and Finite-time Pinching Singularities. Our approach is related to several recent studies on the formation of topological singularities in interfacial flows. These have concerned singularity formation in Stokes flows, in Hele-Shaw flows, and in axisymmetric jets. We discuss two of these situations to illustrate how surface tension can enter the problem in different ways, depending on the assumptions made on the physics.

In the first situation, consider the flow within a Hele-Shaw cell of a long, symmetric neck of fluid surrounded by a gas at constant pressure. The gap-averaged velocity is given by a two-dimensional Darcy's law. A longwave or "lubrication" approximation then gives that the long neck of fluid is governed by

$$
h_{t}=-\frac{b^{2} \tau}{12 \mu}\left(h h_{x x x}\right)_{x}
$$


where $2 h$ is the neck width, $x$ is the coordinate along the length of the neck, $\tau$ is the surface tension parameter, $\mu$ is the fluid viscosity, and $b$ is the gap width of the cell. In this approximation, surface tension introduces a fourth-order degenerate diffusion. Simulations and asymptotics have shown that in a variety of circumstances (choice of initial data, boundary conditions, including large-scale instability) a thin neck can pinch in finite time $[1,12,7,16,17]$. That is, $h\left(x_{c}, t_{c}\right)=0$ at some point $x_{c}$ and time $t_{c}$.

In the second situation, consider an axisymmetric stream of viscous fluid falling under the force of gravity surrounded by a gas of constant pressure. Experiments show that the stream pinches. Dupont \& Eggers [15] have derived a shallow-water approximation that governs the thin neck of fluid that would form before pinching:

$$
\begin{aligned}
\left(h^{2}\right)_{t} & =-\left(v h^{2}\right)_{z} \\
v_{t} & =-v v_{z}-\frac{p_{z}}{\rho}+3 \nu \frac{\left(h^{2} v_{z}\right)_{z}}{h^{2}}-g .
\end{aligned}
$$

Here $z$ is the distance down the neck, $h$ is now the radius of the neck, $v$ is the axial velocity, $g$ is the gravitational constant, $\nu$ is the kinematic viscosity, $\rho$ is the density, and $\tau$ is the surface tension parameter. The pressure jump is proportional to the curvature, which has two components, azimuthal and axial:

$$
p(z)-p_{\text {gas }}=\tau\left(\frac{1}{h \sqrt{1+h_{z}^{2}}}-\frac{h_{z z}}{\left(1+h_{z}^{2}\right)^{\frac{3}{2}}}\right) .
$$

Simulations of this system show finite-time singularities - the stream pinches. This system and related systems have been studied by a number of authors $[8,11,15,14,5,32,41]$. As the stream pinches, simulations show that the azimuthal contribution to the surface tension dominates the axial:

$$
\frac{1}{h \sqrt{1+h_{z}^{2}}} \gg \frac{h_{z z}}{\left(1+h_{z}^{2}\right)^{\frac{3}{2}}} .
$$

In the shallow water expansion, one assumes $h_{z} \ll 1$, hence as the stream begins to pinch, the pressure behaves as $1 / h$. For this reason, Equations (4-5) are effectively a first-order system as the singular time approaches.

In our two dimensional flow, surface tension introduces a behavior much different than in either of these examples. Unlike the second example, there is no azimuthal component of surface tension making the boundary condition on the pressure jump

$$
[p(x)]=-\tau \frac{h_{x x}}{\left(1+h_{x}^{2}\right)^{\frac{3}{2}}} .
$$

This is the term that became negligible in the axi-symmetric flow above. And so, through the pressure gradient $p_{x}$ the surface tension induces a higher-order effect in two dimensions than in the axi-symmetric flow. That this effect is linearly dispersive makes its contribution much different than that in the Hele-Shaw flow.

\section{Full Equations of Motion}

Consider two irrotational, inviscid, incompressible, density-matched fluids separated by time-dependent interfaces $\Gamma_{21}(t)$ and $\Gamma_{12}(t)$. As shown in Figure 3, the fluids are in a horizontal jet configuration - we denote the fluid in the jet by 1 and the fluid above and below this fluid by $2 . \hat{n}$ and $\hat{s}$ are the unit normal and unit tangent vectors to $\Gamma_{i j}(t)$, and $\mathcal{K}_{i j}$ is the curvature. Away from the interface, the fluid velocities, $\overrightarrow{u_{1}}$ and $\overrightarrow{u_{2}}$, satisfy the incompressible Euler's equation

$$
\vec{u}_{j t}+\left(\vec{u}_{j} \cdot \nabla\right) \vec{u}_{j}=-\frac{1}{\rho_{j}} \nabla\left(p_{j}+g y\right), \quad \nabla \cdot \vec{u}_{j}=0 .
$$




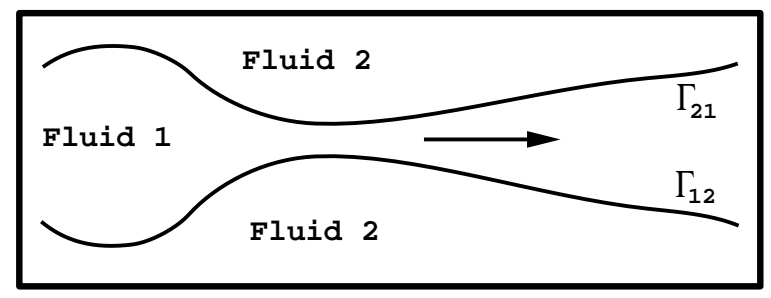

Figure 3. A schematic of the fluid interface problem.

The boundary conditions are

$$
\begin{array}{cl}
{[\vec{u}]_{\Gamma_{i j}} \cdot \hat{n}=0} & \text { kinematic boundary condition } \\
{[p]_{\Gamma_{i j}}=\tau \mathcal{K}_{i j}} & \text { Young-Laplace boundary condition } \\
\vec{u}_{j} \rightarrow(0,0) \quad \text { as } \quad|y| \rightarrow \infty & \text { far-field boundary condition }
\end{array}
$$

There is an additional condition on the velocity of the fluid inside the jet - the velocity has some characteristic speed $v_{c}$. Furthermore, the interface moves with the fluid in the normal direction. In the above, $[\cdot]$ represents the jump from fluid 2 to fluid 1 . The Weber number, We, is a dimensionless quantity that relates the strength of the destabilizing shear (equal to $v_{c}$ since there is no flow at infinity) and the regularizing surface tension:

$$
W e=\frac{\rho \lambda v_{c}^{2}}{\tau} .
$$

$\tau$ is the surface tension parameter and $\lambda$ is the periodicity length. We have assumed the fluids are density matched: $\rho=\rho_{i}$.

In the following, we consider symmetric jets and further assume the interfaces bounding the symmetric jet are the graphs of a function $h:(x, \pm h(x, t))$. Using the kinematic boundary condition and the incompressibility of the fluids, we write the evolution equation for $h$ as a mass conservation law in shallow water form:

$$
h_{t}(x, t)=-\frac{\partial}{\partial x}\left(h(x, t) \frac{1}{h(x, t)} \int_{0}^{h(x, t)} u(x, y, t) d y\right)=-\frac{\partial}{\partial x}(h(x, t) U(x, t))
$$

where $\vec{u}_{1}=(u, v)$ and $U(x, t)$ is the vertical average of the horizontal velocity across the jet.

This choice of an Eulerian parametrization limits what Weber numbers we consider. Figure 2 shows simulations of a symmetric jet with surface tension for two values of the Weber number. These simulations used a different parametrization of the vortex sheets, one that does not assume them to be the graph of a function. The bottom plot in Figure 2 shows a simulation in which the Weber number is small and there is only one unstable length-scale. In this case, the symmetric jet pinches very cleanly [21]. The top plot in Figure 2 shows a simulation with a larger Weber number, one for which there are a number of unstable length-scales. The jet folds over on itself near the pinch [27]. These simulations of the full problem suggest that if the physical parameters are chosen so that the problem has too many unstable length-scales, the interface will try to fold over, causing a finite-time shock. This would be an unphysical singularity, an artifact of our choice of parametrization. 
In Appendix A, we give a reformulation of the boundary integral formulation for the motion of a $2 \pi$-periodic symmetric jet:

$$
\begin{aligned}
h_{t}= & -\frac{\partial}{\partial x}\left(h(x, t) \frac{1}{2 \pi} \int_{0}^{2 \pi} \gamma\left(x^{\prime}, t\right) d x^{\prime}\right. \\
& \left.+\sum_{k=-\infty k \neq 0}^{\infty}\left[\frac{1}{k} \sinh (k h(x, t)) \frac{1}{2 \pi} \int_{0}^{2 \pi} \gamma\left(x^{\prime}, t\right) e^{-|k| h\left(x^{\prime}, t\right)} e^{-i k x^{\prime}} d x^{\prime}\right] e^{i k x}\right) \\
\gamma_{t}= & -(\gamma \tilde{u})_{x}+W e^{-1} \mathcal{K}_{x}
\end{aligned}
$$

where

$$
\begin{aligned}
\tilde{u}(x, t)= & \frac{1}{2} \sum_{k=-\infty}^{\infty} \cosh (k h(x, t))\left(\frac{1}{2 \pi} \int_{0}^{2 \pi} \gamma\left(x^{\prime}, t\right) e^{-|k| h\left(x^{\prime}, t\right)} e^{-i k x^{\prime}} d x^{\prime}\right) e^{i k x} \\
& -\frac{1}{2} \sum_{k=-\infty}^{\infty} \operatorname{sgn}(k) e^{-|k| h(x, t)}\left(\frac{1}{2 \pi} \int_{0}^{2 \pi} \gamma\left(x^{\prime}, t\right) \sinh \left(k h\left(x^{\prime}, t\right)\right) e^{-i k x^{\prime}} d x^{\prime}\right) e^{i k x} .
\end{aligned}
$$

This reformulation is of interest as it is a Fourier series representation, rather than a boundary integral representation. We have not found this reformulation in the literature. In Section 3 we use the reformulation to find an asymptotic model for thin jets. The advantage of the reformulation over the boundary integral formulation is that the necessary asymptotic expansions are much more direct.

In the above, $\gamma$ is the unnormalized sheet strength which is related to the velocity jump in the tangential direction across the upper vortex sheet:

$$
\frac{\gamma(x, t)}{\sqrt{1+h_{x}^{2}}}=-[\vec{u}]_{\Gamma_{21}(p, t)} \cdot \hat{s}=\left(\vec{u}_{1}\left(\Gamma_{21}(x, t), t\right)-\vec{u}_{2}\left(\Gamma_{21}(x, t), t\right)\right) \cdot \hat{s} .
$$

If the surface tension is non-zero $(W e<\infty)$, a smooth solution of the system $(57,58-59)$ conserves the mass, circulation, and its $y$-moment:

$$
\int_{0}^{2 \pi} h(x, t) d x, \quad \int_{0}^{2 \pi} \gamma(x, t) d x, \quad \text { and } \int_{0}^{2 \pi} h(x, t) \gamma(x, t) d x .
$$

Using the boundary integral formulation (see Appendix A), we note that by introducing a new variable, $\eta$ with $\eta_{x}=\gamma$, the system $(57,58-59)$ can be re-written in a Hamiltonian form:

$$
h_{t}=\frac{\delta \mathcal{E}}{\delta \eta} \quad \eta_{t}=-\frac{\delta \mathcal{E}}{\delta h},
$$

where the conserved energy is:

$$
\begin{aligned}
\mathcal{E}(h, \gamma)=W e^{-1} & \left(\int_{0}^{2 \pi} \sqrt{1+h_{x}^{2}} d x-2 \pi\right) \\
& +\frac{1}{8 \pi} \int_{0}^{2 \pi} d x \gamma(x, t) \int_{-\infty}^{\infty} d x^{\prime} \gamma\left(x^{\prime}, t\right) \ln \left(\frac{\left(x-x^{\prime}\right)^{2}+\left(h(x, t)+h\left(x^{\prime}, t\right)\right)^{2}}{\left(x-x^{\prime}\right)^{2}+\left(h(x, t)-h\left(x^{\prime}, t\right)\right)^{2}}\right),
\end{aligned}
$$

This is similar to Zakharov's Hamiltonian formulation based on Luke's variational formulation for water waves, where the Hamiltonian is a function of the surface height and the velocity potential [28, 43]. The local problem presented in Section 4 has a similar Hamiltonian formulation. We do not take much advantage of the Hamiltonian formulation in either case.

The energy is a sum of two positive terms, the line energy and kinetic energy. Energy conservation gives a uniform bound on the interface length $\int \sqrt{1+h_{x}^{2}} d x$ in the positive surface tension $(W e<\infty)$ case. Since the vortex sheets are 1-dimensional, this yields a further bound on $\|h\|_{\infty}$, excluding the possibility of the solution becoming singular by the jet becoming infinitely wide at some point. This is to be contrasted with the case 
of a single vortex sheet with nonzero mean circulation, which has infinite kinetic energy but for which a finite unsigned part is conserved. In this case, there would be no control of the length (see [21]).

We linearize the boundary integral formulation of a periodic jet in the Lagrangian frame, $(57,58-59)$, about the flat steady state solution $x(p, t)=p+\bar{\gamma} t+\epsilon \xi(p, t), y(p, t)=\bar{h}+\epsilon \eta(p, t)$, and $\gamma(p, t)=\bar{\gamma}+\epsilon \mu(p, t)$ with $\epsilon \ll 1$. The linear system has eigenvalues

$$
\sigma_{k}=-i \frac{\bar{\gamma}}{2} k e^{-2 \bar{h}|k|} \pm \frac{1}{2} \sqrt{k^{2}\left(1-e^{-2 \bar{h}|k|}\right)\left(\bar{\gamma}^{2}\left(1+e^{-2 \bar{h}|k|}\right)-2|k| W e^{-1}\right)} .
$$

(The linearization of the reformulated system (12-14) has the same eigenvalues.) There is a band of unstable modes $0<|k|<k_{0}$, where $\bar{\gamma}^{2}\left(1+e^{-2 \bar{h} k_{0}}\right)-2 W e^{-1} k_{0}=0$ and dispersive modes for $|k| \geq k_{0}$. For fixed $k$ and $\bar{h}$ sufficiently large, $e^{-2 \bar{h}|k|} \sim 0$. This corresponds to a very wide jet. In this case, the growth rate (19) becomes

$$
\sigma_{k}= \pm \frac{1}{2} \sqrt{k^{2} \bar{\gamma}^{2}-2|k|^{3} W e^{-1}}
$$

which is the growth rate for a small perturbation of a flat vortex sheet with surface tension. In the zero surface tension case, $W e=\infty$, the growth rate is $\pm \frac{\bar{\gamma}}{2}|k|$, reflecting the catastrophic linear ill-posedness due to the Kelvin-Helmholtz instability. Surface tension dominates this instability for high wave numbers. Such a dispersive regularization has been shown more generally for perturbations of a time-dependent vortex sheet with surface tension [4].

\section{Model Equations for a Thin Jet}

Consider a thin jet, with average height $\bar{h}$ much smaller than the horizontal length-scale $L: \epsilon=\bar{h} / L \ll 1$. We assume $h$ is $O(\epsilon)$ and the sheet strength $\gamma$ is $O(1) . \gamma$ is $O(1)$ through the choice of $v_{c}$ in the definition of the Weber number (10). Writing $h(x, t)=\epsilon H(x, t)$, we expand the equations governing the symmetric jet $(12-14)$ in $\epsilon$. To do this, we need to approximate $e^{-\epsilon|k| H}$. Two options are: a Taylor series approximation

$$
e^{-\epsilon|k| H} \sim 1-\epsilon|k| H+O\left(\epsilon^{2}\right),
$$

or a rational approximation

$$
e^{-\epsilon|k| H} \sim \frac{1}{1+\epsilon|k| H}+O\left(\epsilon^{2}\right)
$$

The Taylor series approximation yields:

$$
\begin{aligned}
\epsilon H_{t} & =-\epsilon(\gamma H)_{x}+\epsilon^{2}\left(H \mathcal{H}\left[(\gamma H)_{x}\right]\right)_{x}+O\left(\epsilon^{3}\right) \\
\gamma_{t} & =-\left(\gamma^{2} / 2\right)_{x}+\epsilon\left(\gamma \mathcal{H}\left[(\gamma H)_{x}\right]\right)_{x}+\epsilon W e^{-1} H_{x x x}+O\left(\epsilon^{2}\right) .
\end{aligned}
$$

Neglecting the higher-order terms yields the asymptotic model

$$
\begin{aligned}
h_{t} & =-(\gamma h)_{x}+\left(h \mathcal{H}\left[(\gamma h)_{x}\right]\right)_{x} \\
\gamma_{t} & =-\left(\gamma^{2} / 2\right)_{x}+\left(\gamma \mathcal{H}\left[(\gamma h)_{x}\right]\right)_{x}+W e^{-1} h_{x x x}
\end{aligned}
$$

where $\mathcal{H}$ is the periodic Hilbert transform

$$
\mathcal{H}[f](x)=\frac{1}{2 \pi} P . V \cdot \int_{0}^{2 \pi} \cot \left(\frac{x-x^{\prime}}{2}\right) f\left(x^{\prime}\right) d x^{\prime} .
$$

This system (22-23) conserves mass, circulation, and the $y$-moment (16), as well as the energy,

$$
2 \mathcal{E}(h, \gamma)=W e^{-1} \int_{0}^{2 \pi} h_{x}(x, t)^{2} d x+\int_{0}^{2 \pi} h\left(\gamma^{2}-\gamma \mathcal{H}\left[(h \gamma)_{x}\right]\right) d x
$$

This energy arises by expansion in $\epsilon$ of the full energy (18) and provides a Hamiltonian formulation (17). 
Unfortunately, this approximate system is linearly ill-posed. Linearizing the asymptotic model (22-23) about the steady solution $h \equiv \bar{h}, \gamma \equiv \bar{\gamma}$, we find the growth-rates

$$
\lambda_{ \pm}(k)=i k\left(-\bar{\gamma}+\bar{\gamma} \bar{h}|k| \pm \sqrt{\bar{h}|k|(\bar{h}|k|-1)\left(\bar{\gamma}^{2}-|k| W e^{-1}\right)}\right) .
$$

The discriminant above tends to negative infinity like $-|k|^{3}$, hence for large $k, \lambda_{-}$has a positive real part, growing like $k^{5 / 2}$. The Taylor series approximation yields an asymptotic model which is even more linearly ill-posed than the jet without surface tension. This occurs because $e^{-\epsilon|k| H} \sim 1-\epsilon|k| H$ is a poor approximation for $\epsilon|k| H \gg 1$. The ill-posedness is unphysical, and is also likely related to the fact that, unlike the full energy, the expanded energy (24) is not strictly signed. We will return to this later in this section.

The asymptotic expansion was made under the assumption that $\epsilon \ll 1$, i.e., the jet is much thinner than the length-scale of the variations of its surface: $\bar{h}|k| \ll 1$. Hence one could argue that the above linear illposedness is not "catastrophic", since we should only consider $1 / \bar{h}$ modes. In this sense, we do not have the unbounded growth rate that causes the difficulties in simulating a single vortex sheet without surface tension. However, as we do not want the numerical simulations to be constrained to consider only $1 / \bar{h}$ modes, we modify the asymptotic model (22-23).

Recalling equation (20),

$$
h_{t}=-(\gamma h)_{x}+O\left(\epsilon^{2}\right)
$$

suggests the following system, the thin jet model:

$$
\begin{aligned}
h_{t}+\left(h \mathcal{H}\left[h_{t}\right]\right)_{x} & =-(\gamma h)_{x} \\
\gamma_{t}+\left(\gamma \mathcal{H}\left[h_{t}\right]\right)_{x} & =-\left(\gamma^{2} / 2\right)_{x}+W e^{-1} h_{x x x} .
\end{aligned}
$$

The two systems (22-23) and (25-26) are equivalent to the full system (12-14) up to terms of $O\left(\epsilon^{2}\right)$. The reader may be familiar with the BBM and $\mathrm{KdV}$ equations, which are related in a similar way [6]. For this reason, it is not surprising that there is loss of conserved quantities. The thin jet model conserves total mass and circulation, but not its $y$-moment. Nor could we find a conserved energy.

Linearizing $(25-26)$ yields the growth rates

$$
\lambda_{ \pm}(k)=\frac{i k}{1+\bar{h}|k|}\left(-\bar{\gamma} \pm \sqrt{\bar{h}|k|\left(W e^{-1}|k|(1+\bar{h}|k|)-\bar{\gamma}^{2}\right)}\right) .
$$

The discriminant above can be negative for an interval of low wave numbers, yielding a band of unstable modes, but is positive for large $k$, yielding a dispersive regularization. Specifically,

$$
|k| \in\left(0, \frac{1}{2 \bar{h}}\left(-1+\sqrt{4 \bar{\gamma}^{2} \bar{h} W e+1}\right)\right) \Longleftrightarrow \lambda_{-}(k) \text { is unstable. }
$$

If the system (25-26) has solutions that become singular in finite time, a natural question is whether the solutions are self-similar. Making the ansatz

$$
h(x, t)=\left(t_{c}-t\right)^{a} H\left(\frac{x-x_{c}}{\left(t_{c}-t\right)^{b}}\right) \quad \gamma(x, t)=\left(t_{c}-t\right)^{c} \Gamma\left(\frac{x-x_{c}}{\left(t_{c}-t\right)^{d}}\right)
$$

and assuming that all the terms in the system (25-26) are of equal order as the singular time is approached, we find that the scaling exponents are completely determined:

$$
h(x, t)=\left(t_{c}-t\right)^{\frac{2}{3}} H\left(\frac{x-x_{c}}{\left(t_{c}-t\right)^{\frac{2}{3}}}\right) \quad \gamma(x, t)=\left(t_{c}-t\right)^{-\frac{1}{3}} \Gamma\left(\frac{x-x_{c}}{\left(t_{c}-t\right)^{\frac{2}{3}}}\right) .
$$

These are the same scaling exponents as for the original symmetric jet problem. These scaling exponents were also suggested by Keller \& Miksis in their study of a thin thread of inviscid fluid surrounded by a trivial flow [23]. They consider the flow with the thread pinching at $t=0$ and study the separation of the regions on either sides of the pinch. We note that the self-similar behavior (29) violates the shallow water formulation 
$h_{x} \ll 1$ since as $t \uparrow t_{c}, h_{x}$ becomes $O(1)$. And while we have determined exponents of self-similarity we leave unaddressed the question of the nature of solutions to the resulting ODEs for $H$ and $\Gamma$.

The rational approximation of $e^{-\epsilon|k| H}$ yields also a linearly well-posed asymptotic model

$$
\begin{aligned}
h_{t} & =-\frac{\partial}{\partial x}\left(h(x, t) \sum_{k=-\infty}^{\infty} \frac{1}{2 \pi} \int_{0}^{2 \pi} \frac{\gamma\left(x^{\prime}, t\right)}{1+|k| h\left(x^{\prime}, t\right)} e^{-i k x^{\prime}} d x^{\prime} e^{i k x}\right) \\
\gamma_{t} & =-(\gamma \tilde{u})_{x}+W e^{-1} h_{x x x}
\end{aligned}
$$

where

$$
\begin{aligned}
\tilde{u}=\frac{1}{2}\left(\sum_{k=-\infty}^{\infty}\right. & \frac{1}{2 \pi} \int_{0}^{2 \pi} \frac{\gamma\left(x^{\prime}, t\right)}{1+|k| h\left(x^{\prime}, t\right)} e^{-i k x^{\prime}} d x^{\prime} e^{i k x} \\
& \left.\quad-\sum_{k=-\infty}^{\infty} \frac{|k|}{1+|k| h(x, t)} \frac{1}{2 \pi} \int_{0}^{2 \pi} \gamma\left(x^{\prime}, t\right) h\left(x^{\prime}, t\right) e^{-i k x^{\prime}} d x^{\prime} e^{i k x}\right) .
\end{aligned}
$$

The expansions yielding (20-21) and (30-32) are discussed in Appendix A.1.

Finally, the Hamiltonian formulation (17) suggests a third asymptotic model. The ill-posed asymptotic model (22-23) conserves the energy (24). This energy is not strictly signed since

$$
-\frac{1}{2} \int_{0}^{2 \pi} f(x) \mathcal{H}\left[f_{x}\right](x) d x=-\frac{1}{\pi} \sum_{l=-\infty}^{\infty}|l|\left|\hat{f}_{l}\right|^{2} .
$$

A small $\epsilon$ expansion of $(1-\epsilon)^{2} \sim 1-2 \epsilon$ has a similar loss of signedness, if one makes the expansion and then do not continue to constrain $\epsilon=\bar{h}|k|$ to be small. For this reason, we make the energy (24) signed by adding an $O\left(\epsilon^{3}\right)$ term $^{1}$ :

$$
2 \mathcal{E}(h, \gamma)=W e^{-1} \int_{0}^{2 \pi} h_{x}(x, t)^{2} d x+\int_{0}^{2 \pi} h\left(\gamma-\frac{1}{2} \mathcal{H}\left[(h \gamma)_{x}\right]\right)^{2} d x
$$

The Hamiltonian system (17) based on this energy yields a linearly well-posed asymptotic model.

The three asymptotic models for a thin jet (25-26), (30-31), and that based on (33), are all equivalent up to order $O\left(\epsilon^{2}\right)$. However, the "thin jet model" $(25-26)$ is the only model which is both linearly ill-posed in the absence of surface tension and has growth rates $\lambda_{k} \sim i k^{3 / 2}$ for $k \gg 1$ in the presence of surface tension. Because of this similarity to the full system, we study the thin jet model extensively in Section 5 . However, we do note that numerical simulations show that all three models readily form pinching singularities.

\section{A Local Model}

Retaining only the lowest-order terms of equations (20-21) gives the purely local system

$$
\begin{aligned}
h_{t}+\gamma h_{x} & =-h \gamma_{x} \\
\gamma_{t}+\gamma \gamma_{x} & =0 .
\end{aligned}
$$

Surface tension and nonlocality enter at the next order. This system is solved exactly by the method of characteristics:

$$
\gamma_{x}(x(\xi, t), t)=\frac{\gamma_{0 x}(\xi)}{1+\gamma_{0 x}(\xi) t} \quad h(x(\xi, t), t)=\frac{h_{0}(\xi)}{1+\gamma_{0 x}(\xi) t}
$$

where $x(\xi, t)=\xi+t \gamma_{0}(\xi)$. As a function of $x$, these solutions have a finite-time singularity where $\gamma$ shocks and $h$ goes to infinity on the characteristic through $\xi_{0}$, the point at which $\gamma_{0 x}$ is the most negative. These

\footnotetext{
${ }^{1}$ The energy (24) is the truncation $\epsilon \mathcal{E}_{1}+\epsilon^{2} \mathcal{E}_{2}$ of the full energy (18): $\mathcal{E}=\epsilon \mathcal{E}_{1}+\epsilon^{2} \mathcal{E}_{2}+\epsilon^{3} \mathcal{E}_{3} \ldots$ The term we add to make the energy signed is not one of the higher-order terms.
} 
solutions provide a simple example of the difficulties of fitting power-law behavior numerically. We discuss this in the Appendix B.

A very interesting system is found by retaining only the surface tension contribution from the next order contributions; that is, the nonlocal terms are neglected. We call this system the local model:

$$
\begin{aligned}
h_{t}+\gamma h_{x} & =-h \gamma_{x} \\
\gamma_{t}+\gamma \gamma_{x} & =W e^{-1} h_{x x x} .
\end{aligned}
$$

While this system is asymptotically inconsistent, we find that nonetheless it produces pinching singularities that are very similar to those we observe in the thin jet model - the interface again forms a corner, and has nearly identical temporal and spatial singularity structure (see Sect. 5.4). Unlike the thin jet model, it retains the Hamiltonian formulation and all the conserved quantities of the full system, and is simple enough that we can make some analytical observations.

In Section 5.3, simulations of the thin jet model (25-26) in the presence of surface tension show a finite-time pinching singularity. As we discuss in Subsection 5.3, the nonlocal terms are not sub-dominant to the surface tension term as the singular time approaches. However, our simulations of the local model (in Section 5.4) show that while its large-scale evolution can be quite different from that of the thin jet model, the fine-scale structure of its pinching singularities are strikingly close to those of the thin jet model. For this reason, we conjecture that a fine analysis of the singularity formation would reveal that the nonlocal terms are slaved to the surface tension term, making the local model of physical interest.

Smooth solutions of the local model, either periodic or on the line with decay at infinity, conserve mass, circulation, and the $y$-moment (16). Solutions also conserve the energy

$$
2 \mathcal{E}(h, \gamma)=W e^{-1} \int h_{x}^{2}(x, t) d x+\int h(x, t) \gamma^{2}(x, t) d x .
$$

The energy (38) corresponds to the sum of line tension and kinetic energy, and the energy gives the local model a Hamiltonian formulation (17).

The system (36-37) is reminiscent of the KdV equation with small dispersion

$$
\gamma+\gamma \gamma_{x}+\epsilon \gamma_{x x x}=0
$$

and it is natural to expect that introducing surface tension adds a dispersive smoothing, preventing the singularities that occur in the $W e=\infty$ case. In fact, this follows immediately from the energy conservation (38). As we are assuming $h_{0}>0$, both terms in the energy are positive as long as the solution remains positive, hence

$$
W e^{-1} \int h_{x}(x, t)^{2} d x \leq M\left(h_{0}, \gamma_{0}\right) \quad \text { and } \quad \int h(x, t) \gamma(x, t)^{2} \leq M\left(h_{0}, \gamma_{0}\right) \quad \forall t \in[0, T] .
$$

On the line, boundedness of the $H^{1}$ norm of $h$ implies that $h$ is uniformly bounded. Hence $h$ cannot go to infinity in finite time, as it must in the $W e=\infty$ case. In short, if there is a finite-time singularity for the $W e<\infty$ case it is of a different type than the $W e=\infty$ singularity. In the case where $h_{0}>0$ and $\gamma_{0}$ are smooth, we conjecture that solutions are smooth on any time interval $[0, T]$ on which $h$ remains strictly positive, and that singularities arise only when $h$ becomes zero at a point.

While we are concerned with the problem of a thin jet pinching in two, for which the initial $h_{0}$ is positive, a finite-time singularity must happen in the case of different initial data:

Theorem. If $W e<\infty$, and $h_{0}$ and $\gamma_{0}$ are both smooth on $\mathbb{R}$ and compactly supported in the interval $[a, b]$ then the solution to (36-37) must lose smoothness in finite time.

This theorem could apply to the post-pinch situation if after the change of topology there are thin bubbles with small slopes. The theorem is proved by showing that smooth solutions satisfy a variance identity:

$$
\frac{d^{2}}{d t^{2}} \int_{a}^{b} x^{2} h(x, t) d x=2 \int_{a}^{b} h \gamma^{2}+3 W e^{-1} \int_{a}^{b} h_{x}^{2} \geq 4 \mathcal{E}(h, \gamma)=4 \mathcal{E}\left(h_{0}, \gamma_{0}\right) .
$$


The energy provides an upper bound on the variance, hence

$$
c_{1}+c_{2} t+2 \mathcal{E}\left(h_{0}, \gamma_{0}\right) t^{2} \leq \int_{a}^{b} x^{2} h(x, t) d x \leq C\left(h_{0}, \gamma_{0}\right)
$$

determines an upper bound on the time of existence for smooth solutions.

For such initial data, Sideris has a blow-up argument based on $\int x \gamma(x, t) d x$. The proof is a generalization of a convexity argument for the inviscid Burgers equation that shows that if solutions remain smooth and if $\int x \gamma_{0}(x) d x>0$, then this first moment must blow up in finite time [38]. There are analogous blow-up results for periodic solutions to (36-37) if both $h_{0}$ and $\gamma_{0}$ vanish in some sub-interval of the periodic domain.

As the simulations of the local model in Section 5.4 show finite-time pinching singularities, we look for self-similar solutions of the form:

$$
h(x, t)=\left(t_{c}-t\right)^{a} H\left(\frac{x-x_{c}}{\left(t_{c}-t\right)^{b}}\right) \quad \gamma(x, t)=\left(t_{c}-t\right)^{c} \Gamma\left(\frac{x-x_{c}}{\left(t_{c}-t\right)^{d}}\right) .
$$

Assuming that all the terms in (36-37) are of equal order as $t \uparrow t_{c}$, we find that the scaling exponents are not completely determined:

$$
h(x, t)=\left(t_{c}-t\right)^{a} H\left(\frac{x-x_{c}}{\left(t_{c}-t\right)^{1 / 2+a / 4}}\right) \quad \gamma(x, t)=\left(t_{c}-t\right)^{a / 4-1 / 2} \Gamma\left(\frac{x-x_{c}}{\left(t_{c}-t\right)^{1 / 2+a / 4}}\right) .
$$

This is an expected difference between the local model and the thin jet model. The thin jet model (25-26) has additional terms which completely determine the scaling exponents (29).

For self-similar solutions, the coupled system of PDE's becomes a coupled system of ODEs:

$$
\begin{aligned}
4 a H-(2+a) \eta H^{\prime}-4 H \Gamma^{\prime}-4 \Gamma H^{\prime} & =0 \\
(2-a) \Gamma+(2+a) \eta \Gamma^{\prime}+4 \Gamma \Gamma^{\prime}-4 W e^{-1} H^{\prime \prime \prime} & =0
\end{aligned}
$$

where the derivatives are with respect to $\eta=\left(x-x_{c}\right) /\left(t_{c}-t\right)^{1 / 2+a / 4}$.

We have been unable to determine the scaling exponent $a$ from other considerations. We tried matching a self-similar inner solution to a slowly-varying far-field solution. Specifically, we assume $H(\eta) \sim \eta^{\alpha}, \Gamma(\eta) \sim \eta^{\beta}$ for $|\eta| \gg 1$. The far-field spatial exponents $\alpha=4 a /(a+2)$ and $\beta=(a-2) /(a+2)$ are then determined by (39). We found that the ODEs (40-41) did not have the lower-order terms in the equations select an exponent a. There is a similar free exponent in similarity solutions to an axisymmetric Stokes flow [32]. Brenner, Lister, \& Stone [8] have a method that selects a countable number of exponents. We tried to apply their methods but found that since the surface tension term enters with three derivatives, rather than two, we were unable to close the needed recurrence relations.

We can use the energy (38) to find a constraint on $a$, the rate at which $h$ might pinch. If we assume that the self-similar solution matches onto an outer solution at some large, but finite, $\eta_{0}$, then for $t$ close to $t_{c}$

$$
\int_{|x| \leq \eta_{0}\left(t_{c}-t\right)^{1 / 2+a / 4}} h(x, t) \gamma(x, t)^{2} d x \leq \int_{-\pi}^{\pi} h(x, t) \gamma(x, t)^{2} d x \leq M .
$$

Since the solution is self-similar for $|x| \leq\left(t_{c}-t\right)^{1 / 2+a / 4}$,

$$
\begin{aligned}
\left(t_{c}-t\right)^{7 a / 4-1 / 2} \int_{|\eta| \leq 1} H(\eta) \Gamma(\eta)^{2} d \eta & =\int_{|x| \leq\left(t_{c}-t\right)^{1 / 2+a / 4}} h(x, t) \gamma(x, t)^{2} d x \\
& \leq M .
\end{aligned}
$$

This implies $7 a / 4-1 / 2 \geq 0$ hence $a \geq 2 / 7$.

Finally, as pointed out by D. McLaughlin, the local model does have traveling wave solutions where $h>0$, but none where $h$ is zero at points [29]. Assuming $h(x, t)=H(x+c t)=H(y)$ and $\gamma(x, t)=\Gamma(x+c t)=\Gamma(y)$, 
we find a coupled system of ODEs:

$$
\begin{aligned}
c H^{\prime}(y)+(H(y) \Gamma(y))^{\prime} & =0 \\
c \Gamma^{\prime}(y)+\frac{1}{2}\left(\Gamma(y)^{2}\right)^{\prime} & =W e^{-1} H^{\prime \prime \prime}(y) .
\end{aligned}
$$

Integrating with respect to $\mathrm{y}$,

$$
\begin{aligned}
c H(y)+H(y) \Gamma(y) & =A_{1} \\
c \Gamma(y)+\frac{1}{2} \Gamma(y)^{2} & =W e^{-1} H^{\prime \prime}(y)+A_{2} .
\end{aligned}
$$

Solving (42) for $\Gamma(y)$, equation (43) can be viewed as a particle in a potential:

$$
H^{\prime \prime}(y)=W e\left(\frac{A_{1}^{2}}{2 H(y)^{2}}-A_{2}-\frac{c^{2}}{2}\right)=-W e \frac{d}{d H} \phi(H)
$$

where $\phi(H)=\left(A_{2}+c^{2} / 2\right) H+A_{1}^{2} /(2 H)$. This potential clearly requires profiles $H$ be strictly positive, and has a minimum if $A_{1} \neq 0$ and $A_{2}+c^{2} / 2>0$. Traveling wave solutions correspond to orbits whose period divides $2 \pi$.

\section{Numerical Results}

\subsection{Numerical methods.}

To compute $2 \pi$-periodic solutions of the thin jet model,

$$
\begin{aligned}
h_{t}+\left(h \mathcal{H}\left[h_{t}\right]\right)_{x} & =-(\gamma h)_{x} \\
\gamma_{t}+\left(\gamma \mathcal{H}\left[h_{t}\right]\right)_{x} & =-\left(\gamma^{2} / 2\right)_{x}+W e^{-1} h_{x x x},
\end{aligned}
$$

we uniformly discretize the interval with $n$ grid points. Derivatives and Hilbert transforms are calculated by discrete Fourier transforms, and nonlinearities are evaluated by pseudo-spectral collocation.

We first solve equation (44) for $h_{t}$. Since $h_{t}$ has zero mean, its anti-derivative, $Z$, is a periodic function and equation (44) is equivalent to

$$
\mathcal{N}(Z)=\frac{1}{h} Z+\partial_{x} \mathcal{H}[Z]=-\gamma
$$

where $\mathcal{N}$ is symmetric positive definite operator. $Z$ is solved for by conjugate gradient iteration using spectral preconditioning. Extrapolation from previous solutions provides a good first guess for the iteration. We use a stopping tolerance of $10^{-18}$ - this tolerance was always met within $20-40$ iterations. Differentiation of $Z$ yields $h_{t}$, which then determines $\gamma_{t}$ from equation (45).

Once $h_{t}$ and $\gamma_{t}$ are known, a fourth-order Adams-Bashforth scheme is used for the time-stepping. We find that for $n$ mesh-points in $[0,2 \pi]$, there is a stability constraint on the size of the time-step in that the high $k$ modes of the spatial Fourier transform will grow if time-steps are too large. We find that satisfying this stability constraint is sufficient for very high accuracy in the time-stepping. Larger time-steps can be taken stably using a Runge-Kutta scheme, however the increase in step-size was not found to be large enough to compensate for its relative inefficiency.

As the active part of the spectrum - those modes whose amplitudes are above the level of round-off error - approaches the Nyquist frequency (the $n / 2$ mode), we avoid loss of resolution by stopping the simulation and doubling the number of mesh-points ${ }^{2}$.

The numerical method conserves $\int h$ and $\int \gamma$ automatically. As the thin jet model does not have any other conserved quantities, we cannot use them to monitor accuracy. We do, however, check that we have $O\left(\Delta t^{4}\right)$ pointwise convergence at late times in the simulation. As an additional check of the correctness of the code,

\footnotetext{
${ }^{2}$ The point-doubling is done by taking the solution at $n$ points, computing its Fourier transform, and extending the Fourier transform from $n / 2$ modes to $n$ modes by defining the new modes to have zero Fourier amplitude. The reverse Fourier transform then yields a solution at $2 n$ points.
} 
we take initial data which is an $\epsilon$-perturbation of the constant solution and verify that the computed solution agrees to $O\left(\epsilon^{2}\right)$ with the exact solution of the linearized problem.

The uniform mesh allows us to compute the Hilbert transform quickly and with spectral accuracy. Moreover, the spectrum of the solution can be examined for the spatial structure of the singularity. However, the temporal behavior (e.g., the rate at which $h_{\text {min }}$ goes to zero) might be better studied with an adaptive mesh code, where the grid could be refined near the singular point, allowing the solution to be computed to times closer to the singular time.

To compute solutions of the local model

$$
\begin{aligned}
h_{t} & =-(h \gamma)_{x} \\
\gamma_{t} & =-\frac{1}{2}\left(\gamma^{2}\right)_{x}+W e^{-1} h_{x x x},
\end{aligned}
$$

we use a Crank-Nicolson/Leapfrog pseudo-spectral scheme because of its stability. This is necessary since the linear analysis of the local model shows that the dispersion is of $2^{\text {nd }}$-order - higher than that of the thin jet model. We leap over $\left(\gamma^{2}\right)_{x}$ in equation (47), and Crank-Nicolson the remaining terms:

$$
\begin{aligned}
& \frac{h^{i+1}-h^{i-1}}{2 \Delta t}=-\frac{1}{2}\left(h^{i+1} \gamma^{i-1}\right)_{x}-\frac{1}{2}\left(h^{i-1} \gamma^{i+1}\right)_{x} \\
& \frac{\gamma^{i+1}-\gamma^{i-1}}{2 \Delta t}=-\frac{1}{2}\left(\gamma^{i^{2}}\right)_{x}+\frac{W e^{-1}}{2} h_{x x x}^{i+1}+\frac{W e^{-1}}{2} h_{x x x}^{i-1} .
\end{aligned}
$$

We do not leap over $(h \gamma)_{x}$ in (46) since the linear stability analysis of such a scheme does not suggest a gain in time-step size. We use a GMRES iteration [34] to solve for the solution at the time $i+1$ in terms of the solutions at times $i-1$ and $i$.

\subsection{Simulations of the thin jet and local models with zero total circulation.}

As we discuss in Section 4, the local model (36-37) is similar to the KdV equation with small dispersion in that both problems have a Burgers' shock in the absence of dispersion. We also recall that in a water wave model, Zakharov et al. study singularity formation where the singularity is driven by an inviscid Burgers shock [26].

We study both the thin jet and local models with initial data

$$
h_{0}(x)=0.1+0.05 \cos (x) \quad \gamma_{0}(x)=0.2 \sin (x) .
$$

with Weber number $W e^{-1}=0.005$. This data is chosen so that for the local model in the absence of surface tension, the shock in $\gamma$ and the divergence of $h$ both occur at $x_{c}=\pi$. Both the local and thin jet models preserve the symmetry of this initial data: $h$ is even about $\pi$ and $\gamma$ is odd. Since the initial data has zero total circulation, the thin jet model has no linearly unstable modes.

Figure 4 shows a simulation of the local model (36-37) with this initial data. The evolution of the thin jet model is very similar. As long as $h_{x x x}$ is small, equation (37) is close to the inviscid Burgers equation and $\gamma$ tries to shock. At $\pi$, equation (36) shows that since $\gamma_{x}(\pi, t)$ is decreasing, $h(\pi, t)$ must increase. As $h$ increases, $h_{x x x}$ becomes large, and the surface tension term of equation (37) comes into play. At this point in the evolution, $\gamma$ behaves quite differently than it would in the KdV equation with small dispersion. In the $\mathrm{KdV}$ equation, $\gamma$ tries to shock and the shock is prevented by dispersive waves traveling away from the shock region. In the local model, (36-37), peaks begin to form at the maximum and minimum points of $\gamma$. These peaks do not disperse away; they keep growing. Since equation (36) corresponds to conservation of mass, where $|\gamma|$ is growing $h$ must decrease. Hence $h$ develops a minimum to each side of $\pi$ - these minima decrease to zero as $|\gamma|$ increases to infinity. The fluid is flowing from both sides into a central bubble since $\gamma \uparrow \infty$ on the left and $\gamma \downarrow-\infty$ on the right. The divergences of $\gamma$ satisfy the implication from the shallow water form of equation (36) that $\gamma_{x}$ diverges if $h$ goes to zero.

This mechanism seems to also apply to the thin jet model. Figure 5 shows the final profiles of both the local and thin jet model simulations. One obvious difference between the two profiles is that the nonlocal 

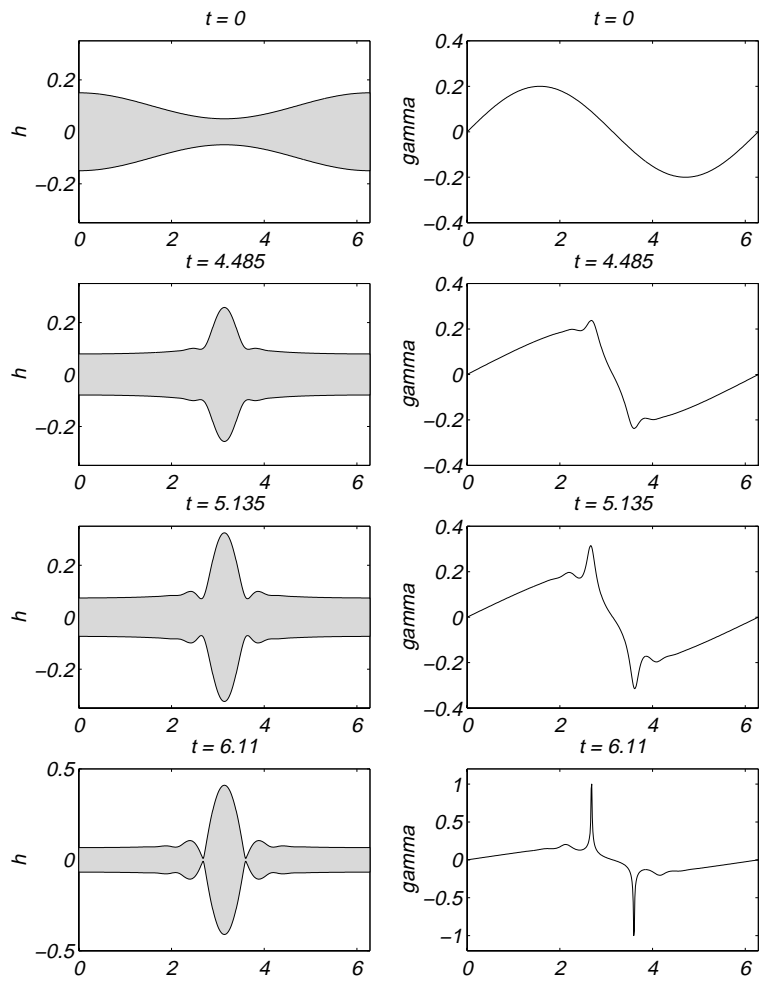

Figure 4. The long-time evolution of the local model. The initial sheet strength $\gamma_{0}$ is chosen to have zero mean so that the shock region is fixed at $x=\pi$.

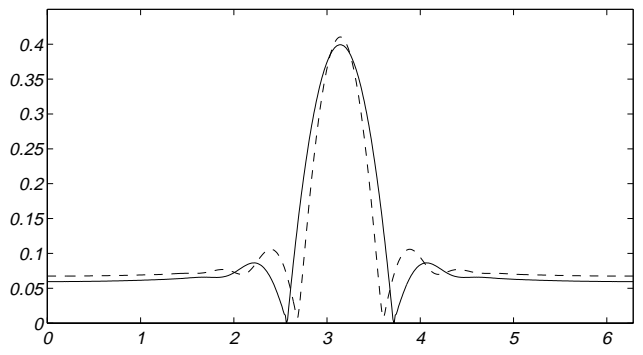

Figure 5. Final states of the local model and the thin jet model where the both solutions have the same initial data. Dashed line: local model, singular time $t=6.11$. Solid line: thin jet model, singular time $t=7.68$.

terms appear to introduce some smoothing: the thin-jet model solution does not have the noticeable ripple near the singularity that the local model solution does.

We will not examine these particular simulations in any further detail. In the next section, to gain information on the spatial form of oncoming singularities, we will carefully examine the behavior of the spatial Fourier spectrum. However, this is done far more easily when a single singularity forms in the period.

In the thin jet model we induce the formation of a single pinching singularity by considering initial data $\gamma_{0}$ with nonzero mean $\bar{\gamma}$. Unlike the local model, the effect of $\bar{\gamma}$ cannot be removed through a change of frame. We further choose $h_{0}$ so that the local model also pinches at only one point. In all of our simulations where 
the jet pinches at a single point, $\gamma$ diverges. We have not found any initial data which lead to $\gamma_{x}$ diverging but $\gamma$ remaining bounded.

\subsection{Simulations of the thin jet model with nonzero total circulation.}

In this section, we analyze the spatial and temporal structure of finite-time pinching singularities in the thin jet model, choosing initial data such that $h$ pinches at a single point in $[0,2 \pi]$.

For $\bar{\gamma} \neq 0$, the thin jet model can have linearly unstable modes. Figure 2 shows simulations of a full symmetric jet which suggest that if the physical problem has too many unstable length-scales, the jet may fold over on itself [27]. This would cause our model to shock. To avoid this unphysical singularity, we use the linear stability analysis in Section 3 to choose $W e$, so that the unstable band (28) contains only the $k=1$ mode, and take initial data

$$
\left(h_{0}(x), \gamma_{0}(x)\right)=\left(\bar{h}+\epsilon h_{1}(x), \bar{\gamma}+\epsilon \gamma_{1}(x)\right) \quad \epsilon \ll 1
$$

where $\left(h_{1}, \gamma_{1}\right)$ is the corresponding unstable eigenfunction of the linearized system.
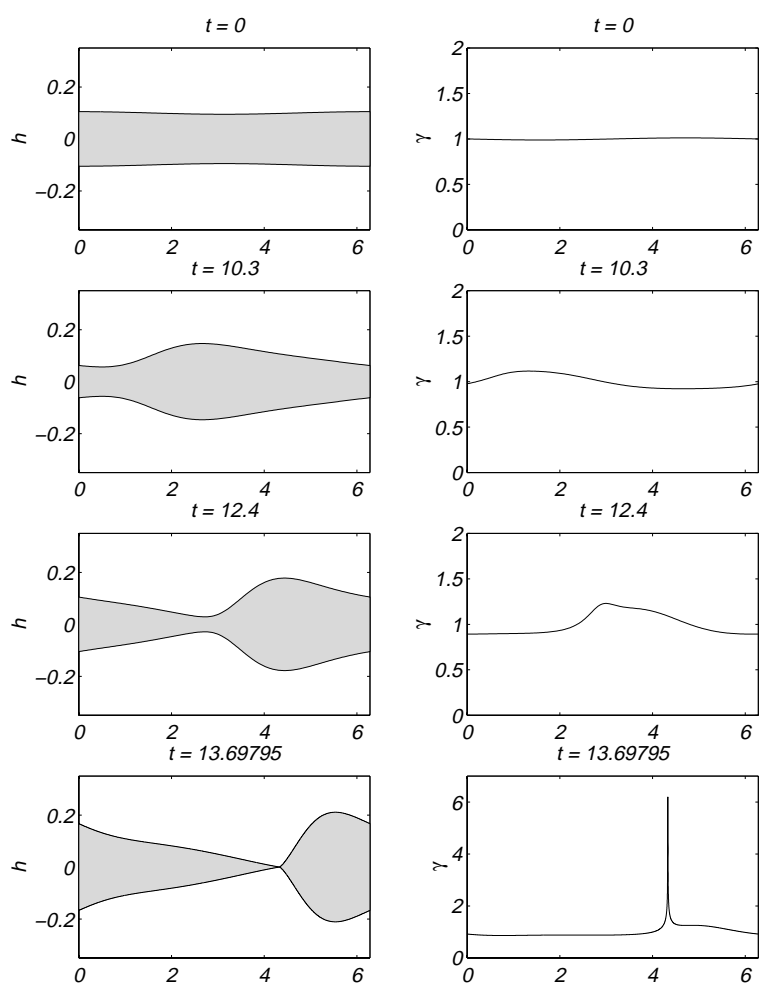

Figure 6 . The long-time evolution of the thin jet model from unstable eigenfunction initial data.

Figure 6 shows the simulation of the thin jet model $(25-26)$, with $W e^{-1}=0.435=\bar{\gamma}^{2} /(2+3 \bar{h})$ and initial data

$$
h_{0}(x)=0.1(1+0.05 \cos (x)) \quad \gamma_{0}(x)=1-0.01142 \sin (x) .
$$

Since $\bar{\gamma}>0$, the maxima and minima of $h$ and $\gamma$ move to the right, passing through a number of periods, before $\gamma$ starts to form a noticeable localized peak. Near $\gamma$ 's peak, $h$ has a minimum which begins to decrease to zero. The two extrema, where $\gamma$ achieves its maximum and $h$ achieves its minimum, occur at different points, with these points moving toward each other as the solution evolves. This behavior continues, $\gamma$ 's maximum becoming larger and more pointed as $h$ 's minimum decreases to zero. 
And so, the jet pinches with the velocity in the collapsing neck diverging to infinity. Figure 7 shows the evolution of $h_{x}$. It grows, but does not appear to increase to infinity. The graph of $h_{x}$ gives visual evidence for a corner singularity for $h$ since its slope appears to jump. Details of the spatial structure will be studied through the Fourier spectrum of the solution.
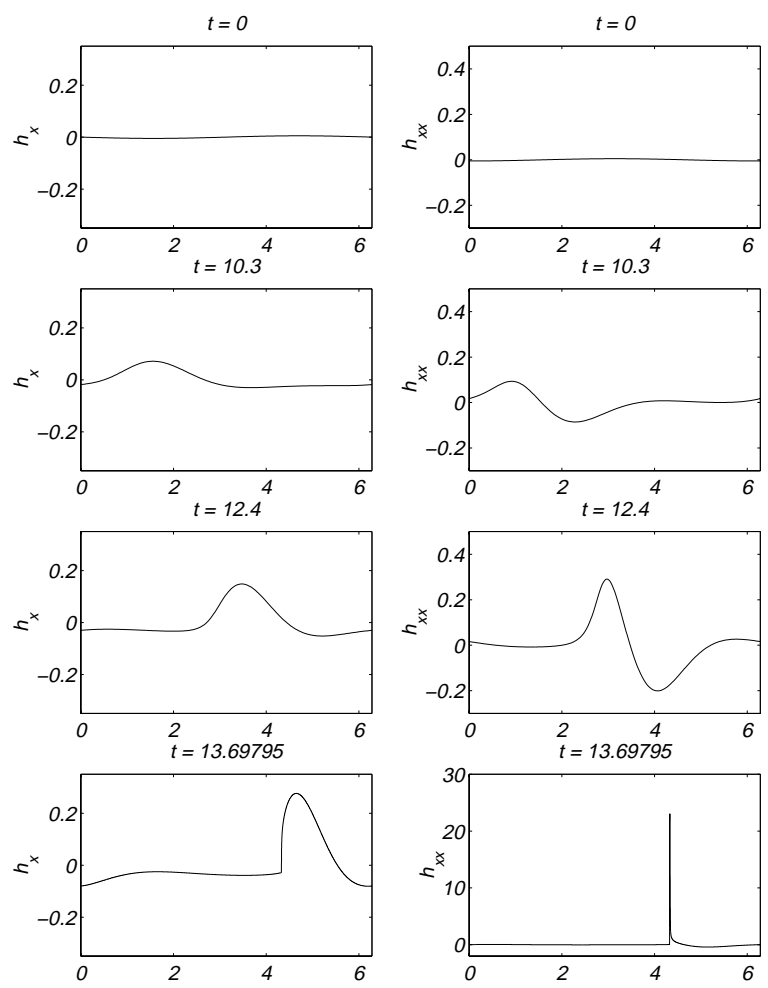

Figure 7. The evolution of $h_{x}$ and $h_{x x}$ for the evolution shown in Figure 6.

We also considered whether any of the terms of the thin jet model became sub-dominant as the singular time was approached. To do this we plotted the $L^{\infty}$ norm of each term of the $\gamma_{t}$ equation against $\log \left(t_{c}-t\right)$, at times late in the simulation. All terms appeared to have the same temporal behavior as $t \rightarrow t_{c}$, suggesting that the thin jet model cannot be simplified by dropping any sub-dominant terms.

We want to quantify the temporal behavior of these collapsing and diverging quantities. In Section 3, we consider self-similar solutions of (25-26). Periodic solutions will be at best locally self-similar, with higherorder corrections, and so one might expect the extrema to have the leading-order behavior

$$
h_{\min }(t) \sim\left(t_{c}-t\right)^{\frac{2}{3}}, \quad \gamma_{\max }(t) \sim\left(t_{c}-t\right)^{-\frac{1}{3}}, \quad h_{x x \max }(t) \sim\left(t_{c}-t\right)^{-\frac{2}{3}} .
$$

As a first attempt to address this expectation, we fit the extrema to a single power law. To do this for the minimum value of $h, h_{\min }\left(t_{i}\right)$ is first found by fitting $h$ to a Fourier polynomial and finding its minimum by Newton's method. We then take three minima, $\left(t_{i-1}, h_{\min }\left(t_{i-1}\right)\right),\left(t_{i}, h_{\min }\left(t_{i}\right)\right)$, and $\left(t_{i+1}, h_{\min }\left(t_{i+1}\right)\right)$, and fit them to $a\left(t_{c}-t\right)^{p}$ by minimizing

$$
\sum_{j=i-1}^{i+1}\left(h_{\min }\left(t_{j}\right)-a\left(t_{c}-t_{j}\right)^{p}\right)^{2}
$$

to determine the amplitude $a$, singular time $t_{c}$, and temporal exponent $p$. The 3-point fit is found for all successive data triples, and one then plots $\left(t_{i}, t_{c}\left(t_{i}\right)\right)$ and $\left(t_{i}, p\left(t_{i}\right)\right)$. If $h_{\min }(t)$ is exactly a power law, then 
these plots will be flat lines since $t_{c}\left(t_{i}\right)$ and $p\left(t_{i}\right)$ are constant. If the power-law behavior is only apparent as $t_{i}$ approaches the singular time $t_{c}$, then the plotted curves should level off as $t_{i} \uparrow t_{c}$. In Appendix B we present an example where the leading-order behavior is known exactly from theoretical considerations. However, in the example, higher-order contributions make it difficult to determine the leading-order behavior from the data using the above method.
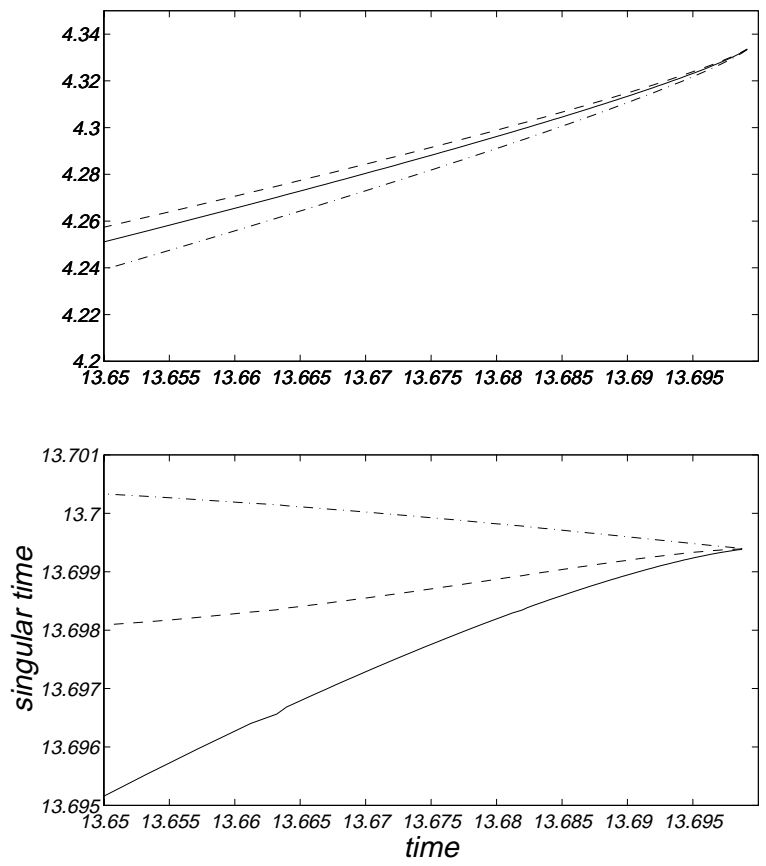

Figure 8. Top: The evolution of the position of extrema for the thin jet model with nonzero surface tension. The $x$-axis is time, the $y$-axis is the position of the extrema. Bottom: The evolution of the fitted singular time from three-point fittings for the thin jet model with nonzero surface tension. The $x$-axis is time, the $y$-axis is the fitted singular time. For both figures, the dashed line is for $\max \left\{h_{x x}\right\}$, the solid line for $\max \{\gamma\}$, and the dot-dashed line for $\min \{h\}$.

We do use three-point fits to argue that a pinching singularity occurs at a finite time. The bottom plot in Figure 8 shows the singular times found from fitting $h_{\min }(t), \gamma_{\max }(t)$, and $h_{x x \max }(t)$ to power laws, plotted as functions of the fitting time, e.g. $\left(t, t_{c}(t)\right)$ for $h_{\text {min }}$. Since there is a single well-defined $t_{c}$ to which the fitted singular times are converging, this figure verifies that $h$ touches down at the same time that $\gamma$ diverges. The top plot in Figure 8 shows the spatial position of each extremum. It shows that although the extrema occur at different points, the points are moving towards each other as $t \uparrow t_{c}$. Taken together, the figures provide strong evidence for the finite-time singularity being of the type:

$$
h\left(x_{c}, t_{c}\right)=0 \quad \gamma\left(x_{c}, t_{c}\right)=\infty \quad h_{x x}\left(x_{c}, t_{c}\right)=\infty .
$$

While the temporal exponents from the three-point fitting have the correct signs, they do not tend to any clear value as the singular time is approached. In Appendix B, we present an approach to data-fitting in which we systematically include the effects of higher-order algebraic corrections to a simple power law. In the top plot of Figure 9, we present the results of both this new method and the 3-point method in fitting $\gamma_{\max }\left(t_{i}\right)$. The dot-dash curve is the estimate of the temporal exponent $p$ from the 3-point fitting method, while the solid curve and those around it are estimates found by the new method. The improved fit is quite 
close to $p=-1 / 3$, the value suggested by self-similarity, shown as a dashed line. The fitted singular time $t_{c}\left(t_{i}\right)$ shows a similar marked improvement. The middle and bottom plots in Figure 9 are analogous figures
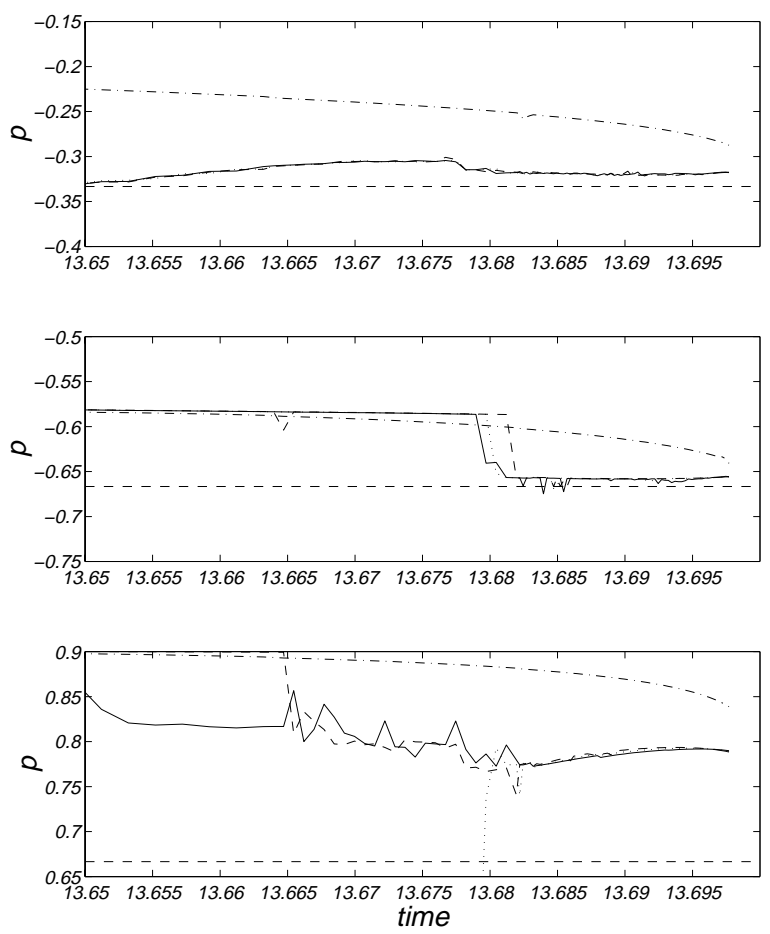

Figure 9. Top: The evolution of the fitted temporal exponent for $\max \{\gamma\}(t)$ for the thin jet model. The dashed line is $p=-1 / 3$. The dot-dash line is the exponent found by threepoint fittings. The remaining lines are from the fitting method described in Appendix B with weights $\alpha=.5, .75,1.0$. Middle: The evolution of the fitted temporal exponent for $\max \left\{h_{x x}\right\}(t)$ for the thin jet model. The dashed line is $p=-2 / 3$. The dot-dash line is the exponent found by three-point fittings. The remaining lines are from the fitting method described in Appendix B with weights $\alpha=.5, .75,1.0$. Bottom: The evolution of the fitted temporal exponent for $\min \{h\}(t)$ for the thin jet model. The dashed line is $p=2 / 3$. The dot-dash line is the exponent found by three-point fittings. The remaining lines are from the fitting method described in Appendix B with weights $\alpha=.5, .75,1.0$.

for the temporal exponents for $h_{\min }$ and $h_{x x \max }$. These figures suggest

$$
h_{\min }(t) \sim\left(t_{c}-t\right)^{0.8} \quad \gamma_{\max }(t) \sim\left(t_{c}-t\right)^{-0.32} \quad h_{x x \max }(t) \sim\left(t_{c}-t\right)^{-0.66},
$$

with $t_{c}=13.6994$. These are to be compared with the temporal exponents from the self-similarity ansatz $2 / 3,-1 / 3$, and $-2 / 3$ respectively. The exponent for $h_{\min }$ that disagrees most strongly with the prediction of self-similarity. This discrepancy is intriguing and we cannot yet account for it.

We now study the spatial structure of the nascent singularity. As the simulations are periodic and on a uniform mesh, the discrete Fourier transform of the solutions can be analyzed for spatial information. The key tool is Laplace's formula, which describes the asymptotic behavior of the Fourier transform of an analytic function $f$ that has algebraic point singularities off of the real axis. Namely, for $k \gg 1$

$$
f(x) \sim(x-(\xi+i \rho))^{\beta} \Longrightarrow|\hat{f}(k)| \sim C e^{-\rho|k|} \frac{1}{|k|^{\beta+1}} g(1 / k) \quad \text { for } \beta>-1
$$


where $\xi+i \rho$ is the closest such singularity to the real axis, and $g$ is an analytic function with $g(0)=1$. If $f$ is real-valued, such complex point singularities come in conjugate pairs. The "radius of convergence", $\rho$, is found by fitting the power spectrum for exponential decay. If $\rho$ decreases to zero in finite time, then $f$ has lost analyticity and has formed a singularity on the real axis. The algebraic degree of the singularity, $\beta$, is found by fitting the power spectrum for algebraic decay. Using the computed Fourier spectra to determine $\rho$ and $\beta$ has been used to investigate singularity formation in many other systems (see, for example, $[40,33,25,37,10,9]$ ).

If there is more than one complex conjugate pair of singularities, this would be immediately evident in the Fourier spectrum since its decay would be modulated, due to phase interference effects. Figure 10 shows the spectra of the solutions $h$ and $\gamma$. They have no modulation, showing monotonic decay for large $k$ until the round-off level is reached. This suggests that the developing spatial structure might be understood in terms
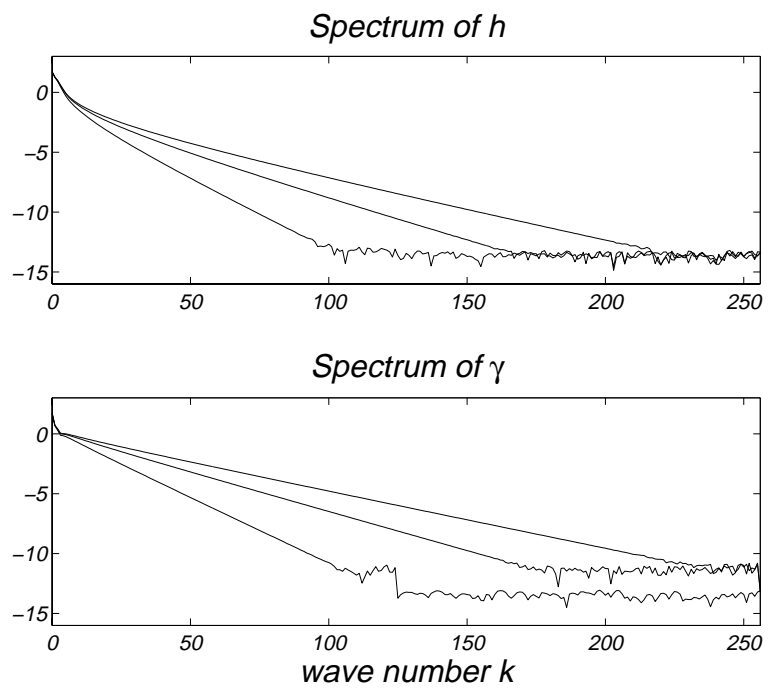

FiguRE 10. Spectra from three different times in the $n=512$ computation of the thin-jet model. The initial spectrum is at the time immediately after point-doubling.

of a single conjugate pair of complex singularities reaching the real axis in finite time. We do find modulated spectra in the solutions with zero total circulation shown in Section 5.2, for which there are two pinching singularities. Fitting spectra with modulation is delicate - it is for this reason we considered solutions that pinch at only one point.

An unmodulated spectrum is fit as follows: given a sequence, $\{|\hat{f}(k)|, \ldots,|\hat{f}(k+L)|\}$, of $L+1$ Fourier amplitudes, we minimize

$$
\frac{1}{L+1} \sum_{i=k}^{k+L}\left(\log (|\hat{f}(i)|)-\log (C)+\rho|i|+(\beta+1) \log (i)-\sum_{j=0}^{m} a_{j} i^{-j}\right)^{2},
$$

to determine $\log (C), \rho, \beta, a_{0} \ldots a_{m}$. Here, we approximate $\log (g(1 / i))$ from (50) with a polynomial of degree $m$. Each stencil of $L+1$ Fourier modes determines one radius of convergence $\rho_{L}(k)$ and one exponent $\beta_{L}(k)$. We then plot $\left(k, \rho_{L}(k)\right)$ and $\left(k, \beta_{L}(k)\right)$. Again, we hope to see that $\rho_{L}(k)$ and $\beta_{L}(k)$ are relatively independent of $k$ for $k \gg 1$.

In this way, we fit for $\rho$ and $\beta$ at a fixed time $t$, and then study their behavior as functions of time. If the singularity corresponds to a complex singularity reaching the real axis in finite time, this would be apparent in $\rho(t): \rho(t) \downarrow 0$ as $t \uparrow t_{c}$. If the algebraic structure of the complex singularity does not change type as the solution becomes singular, then $\beta(t)=\beta\left(t_{c}\right)$ as $t \uparrow t_{c}$. The inviscid Burgers equation provides a simple 
example where the singularity does change type: $\beta(t)=1 / 2$ for $t<t_{c}$, and $\beta\left(t_{c}\right)=1 / 3$. In Appendix $\mathrm{C}$ we discuss the spectral behavior associated with this change in type, as uncovered by the above fitting method.

First the Fourier spectra of $\gamma(x, t)$ is fit at a sequence of times using stencils of different lengths. The top plot of Figure 11 shows $\left(k, \beta_{200}(k)\right)$ for a sequence of times near the singular time. This simulation used $n=16384$ mesh-points; at the first time shown in this figure the solution has about 2000 modes active. In principle, with 2000 active modes and a stencil of length 200 , we should be able to fit 1800 sequences, $\{|\hat{f}(k)| \ldots|\hat{f}(k+200)|\}$. In practice, for a fixed stencil length, the minimization problem becomes ill-conditioned at large wave numbers and we can only fit up to $\beta_{200}(130)$.
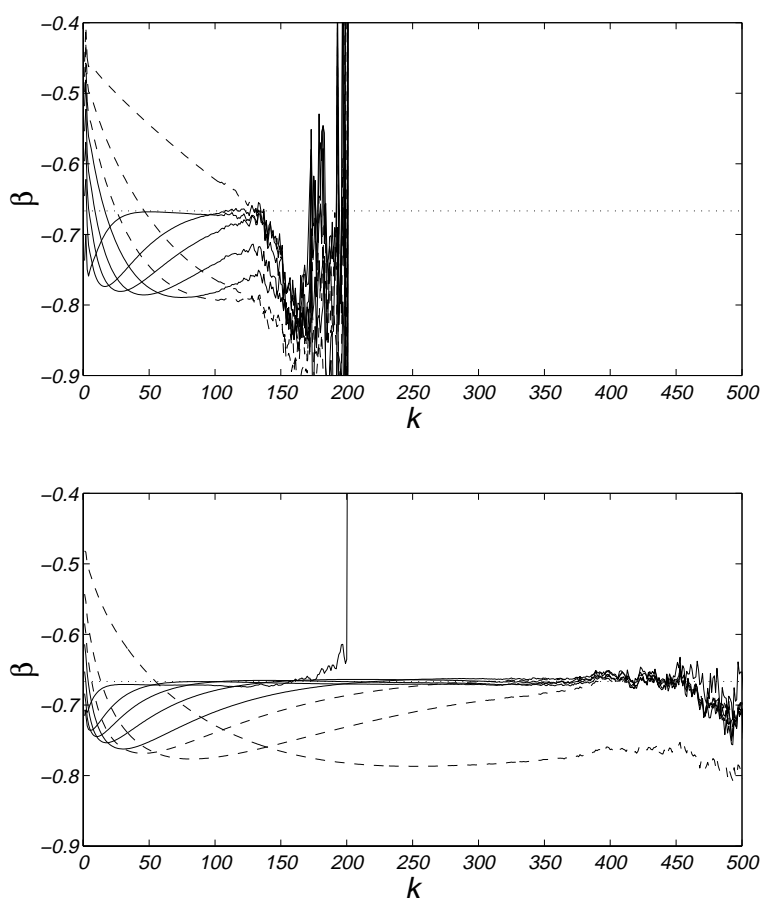

Figure 11. The thin jet model. In both figures, the dotted line marks $\beta=$ $-2 / 3$. The solid lines correspond to times before the active part of the spectrum reached the $n / 2$ mode, the dashed lines for the later times. Top: Using a stencil of length 200 to fit the spectrum of $\gamma$ for spatial structure. Shown at times $t=13.681955,13.693955,13.695955,13.697155,13.697955,13.698355,13.698755,13.699155$.

Bottom: Using a stencil of length 700 to fit $\gamma$ at the same times.

In this graph, the solid lines are for times before $t_{8192}$, the time when the $n / 2=8192$ mode becomes active. The dashed lines are for times after $t_{8192}$. In Appendix $\mathrm{C}$ we demonstrate that aliasing error does not appear to affect the fitting; the time $t_{8192}$ is presented as a lower bound for the time at which the simulation loses accuracy. The horizontal dotted line is $\beta=-2 / 3$. At the earliest time shown, there is a sharp dip downward in $\beta_{200}(k)$ for $k=1 \ldots 40$ and $\beta_{200}(k)$ is nearly constant, approximately $-2 / 3$, for $k=40 \ldots 100$. At the next time shown, the dip has extended to $k=1 \ldots 60$ and $\beta_{200}(k)$ is nearly constant for a smaller number of modes, $k=60 \ldots 100$. This continues, and by the fourth time shown, $\beta_{200}(k)$ does not have any near-constant behavior. For a fixed wave-number $k_{0}, \beta_{200}\left(k_{0}\right)$ initially decreases and then increases.

The bottom plot of Figure 11 is the analogue of the top plot of Figure 11 described above. The longer the stencil, the less ill-conditioned the problem: we can now fit up to $\beta_{700}(400)$. There are two stray curves which are from fitting the first two times - not enough modes were active at these relatively early times. As in the 
top plot of Figure 11, the earliest time has a dip in the low wave-numbers and this dip expands out as time passes. Again, there is a horizontal dotted line at $\beta=-2 / 3$ barely visible at $k$ near 0 and 500 in the figure. This is very strong evidence for $\beta=-2 / 3$ for $k \gg 1$. This is the exponent predicted by Siegel in his study of a Moore's approximation of vortex sheets with surface tension [39].

Comparing the two plots in Figure 11, we see that for a fixed wave number, $k_{0}, \beta_{200}\left(k_{0}\right)$ ranges over more values as the solution evolves than $\beta_{700}\left(k_{0}\right)$ does. This is because the longer the stencil, the smaller the effect of the low wave numbers on the fitting. We could have introduced compensating weights into the least-square fitting. However, while it is clear that for the temporal fittings that times nearest to $t_{c}$ should be emphasized, it is not clear which low wave numbers are in the asymptotic regime described by Laplace's formula (50). Specifically, a natural choice of weights would be $w_{i}=e^{\rho\left(k-k_{c}\right)}$, where $k_{c}$ is the (unknown) wave number past which the asymptotic behavior dominates.

It is possible that the "dip" we see developing and broadening in the low wave-numbers may be the sign of a change of type of the singularity as it approaches the real axis. However, this behavior is quite different from that seen in the example of the inviscid Burgers equation presented in Appendix C.

The top plot of Figure 12 shows the fit $\left(k, \rho_{700}(k)\right)$ at a sequence of times. This plot demonstrates that fitting $\rho_{700}$ is more robust than fitting $\beta_{700}$ : $\beta_{700}$ could only be fit up to $k=400$ while $\rho_{700}$ can be fit past $k=600$. This is simply that the exponential behavior dominates the algebraic behavior and thus is easier to fit. $\rho_{700}$ is nearly constant in the region $k=1-600$ and decreases to zero as $t$ increases to $t_{c}$. The middle plot of Figure 12 shows the radius of convergence as a function of time. We plot $\rho_{700}(50), \rho_{700}(100), \rho_{700}(150)$, and $\rho_{700}(200)$. The four curves lie on top of one another, as suggested by Figure 12, whose curves are nearly flat. The circle indicates $t_{8192}$. The curve is concave down and strongly suggests that the radius of convergence goes to zero in finite time. Fitting this data to a power law gives a singular time of $t_{c}=13.699$, consistent to all given digits with the estimates from the temporal data fits.

Finally, we show the fits of the spectrum of $h$. The bottom plot of Figure 12 for $h$ is the analogue of the bottom plot of Figure 11 for $\gamma$. These fits for $\beta_{700}(k)$ are fairly flat, especially at the earlier times, and so suggest that $h$ does have a complex singularity structure, but with a time-dependent algebraic degree $\beta(t)$. This degree apparently increases in time. This observation is not a consequence of having chosen stencils of the wrong length: the figure for $\beta_{700}$ does not show significantly less variation with time than a figure for $\beta_{200}$. It is striking that $\gamma$ has strong evidence for a particular algebraic degree $\beta=-2 / 3$ and that this does not force $h$ to also have a time-independent algebraic degree of singularity. In fitting $\rho$, we find that $h$ and $\gamma$ have the same radius of convergence.

We note that $\beta\left(t_{c}\right)=1$ would correspond to $h$ developing a corner at the singularity time. $\beta=1$ is plotted as the horizontal dotted line. The behavior seen in the bottom plot of Figure 12 is not inconsistent with a change in type that leads to a corner forming at the singular time $t_{c}$.

As the algebraic behavior should be most easily fitted where the exponential decay is least, an alternate approach would be to fit the small wave numbers for $\beta$. We do this by fixing the stencils to start at $k=20$ and fitting over stencils of varying lengths $|\hat{f}(20)| \ldots|\hat{f}(L)|$ for $\beta_{L}$. In the top plot of Figure 13 we plot $\left(L, \beta_{L}\right)$ where $\beta_{L}$ comes from fitting $\gamma$ in this way with dotted lines at $\beta=-2 / 3,-3 / 4$. The bottom plot of Figure 13 is the analogous figure from fitting $h$. Again, the solid lines correspond to times before $t_{n / 2}$ and the dashed lines are times after $t_{n / 2}$. We fixed the stencil to start at $k=20$, rather than at some lower mode since Figures 11 and 12 show some scruff near $k=1$. These figures suggest that as the singular time approaches, $h$ is developing a corner singularity and $\gamma$ is changing type.

Comment: We also considered whether the dynamics of the thin jet model were modified by replacing the shallow water curvature term, $h_{x x}$, by the full curvature. We found no discernible change, except that the singularity occurred (very) slightly earlier.

\subsection{Simulations of the local model.}

Computing solutions of the local model (36-37) with the initial data (48) from the thin jet simulation, we find that the solution does not become singular. We computed up to time $t=290$, which is 21 times larger 

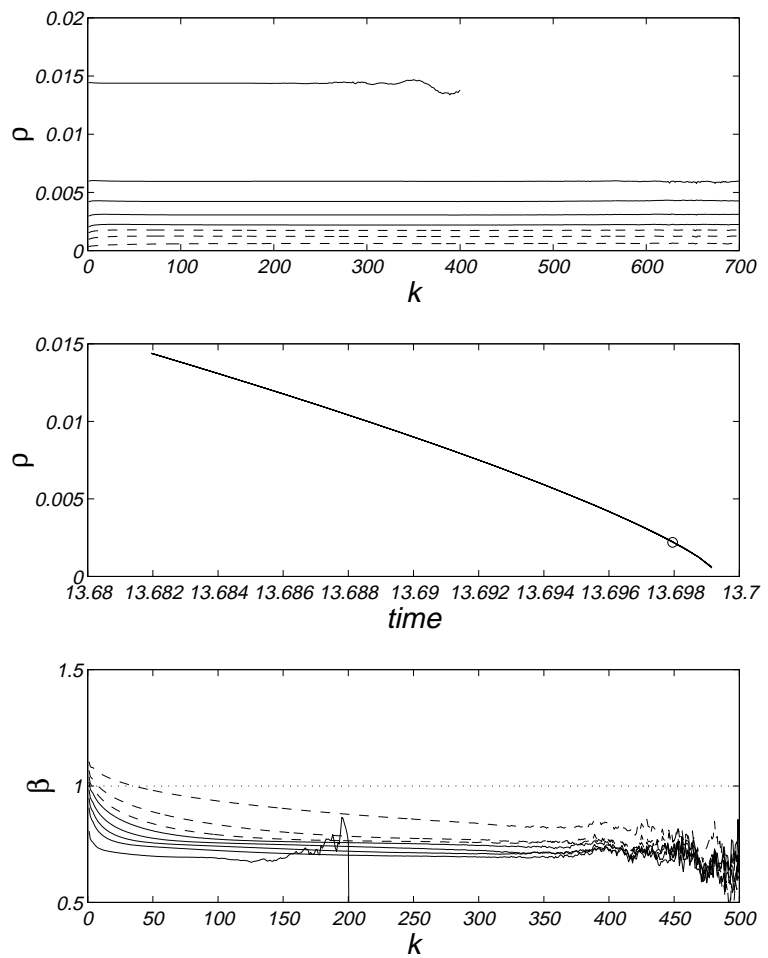

Figure 12. In both the top and bottom figures, the solutions are fitted at the same times shown in Figure 11. The solid lines correspond to times before the active part of the spectrum reached the $n / 2$ mode, the dashed lines for the later times. Top: Using a stencil of length 700 to fit the spectrum of $\gamma$ for a radius of convergence. Middle: The evolution of the radius of convergence for the thin jet model. We plot $\rho_{700}(50), \rho_{700}(100), \rho_{700}(150)$, and $\rho_{700}(200)$ versus time. The circle corresponds to the time at which the active part of the spectrum reached the $n / 2$ mode. Bottom: Using a stencil of length 700 to fit the spectrum of $h$ for spatial structure.

than $t=13.7$, the singular time for the thin jet simulation. Plotting $\left(t, h_{\min }(t)\right)$ and $\left(t, \gamma_{\max }(t)\right)$ for this simulation, we find that the solution appears to be periodic in time.

The thin jet model is different from the local model in that the linearization of the thin jet model can have a band of unstable modes, while the linearization of the local model has solely dispersive modes. For this reason, we cannot take an unstable eigenfunction as initial data for the local model. Taking initial data

$$
h_{0}(x)=0.1+0.05 \cos (x) \quad \gamma_{0}(x)=1.0+0.5 \cos (x)
$$

and $W e^{-1}=0.5$, we compute both the thin jet model (25-26) and the local model (36-37) refining up to $n=8192$ mesh-points. Both simulations have a finite-time singularity of pinching type. Figure 14 shows the evolution of the local model and Figure 15 shows the evolution of the thin jet model. The thin jet model becomes singular at $t_{c} \sim 2.2131$ while the local model becomes singular at $t_{c} \sim 1.8701$.

An immediate difference between the two simulations is that the thin jet model has $\gamma \uparrow \infty$ while the local model has $\gamma \downarrow-\infty$. Considering a range of initial data, we find that the thin jet model always pinches at one point with $\gamma \uparrow \infty$, while the local model can pinch at either one or two points and with either $\gamma \uparrow \infty$ or $\gamma \downarrow-\infty$. 

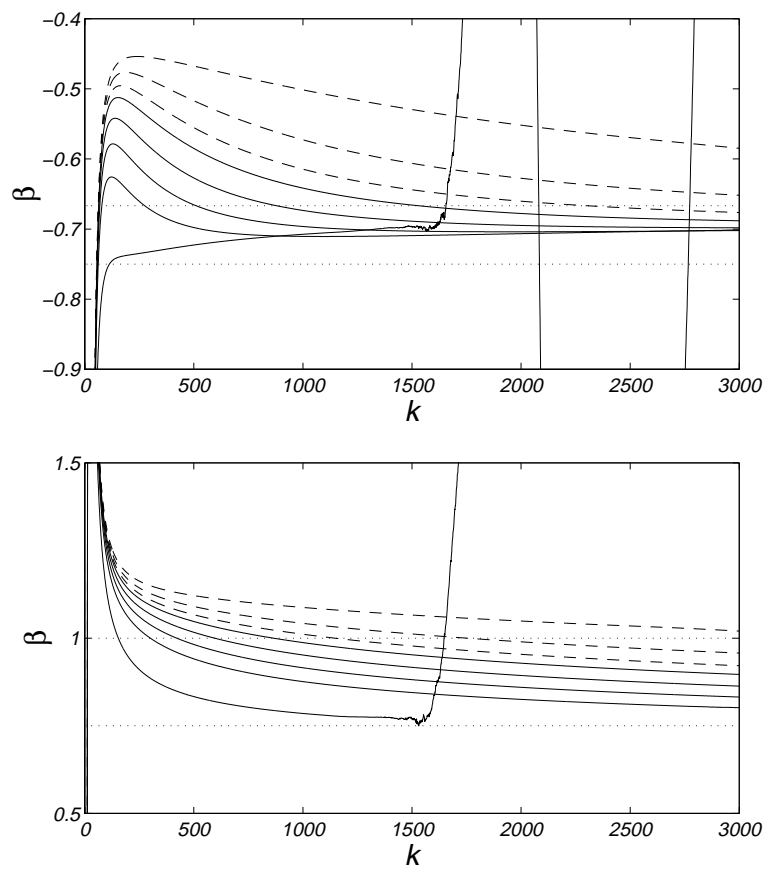

FigURE 13. Using stencils of increasing length to fit the solutions for spatial structure. The solutions are fitted at the same times shown in Figure 11. The solid lines correspond to times before the active part of the spectrum reached the $n / 2$ mode, the dashed lines for the later times. Top: Spectrally fitting $\gamma$ from the thin jet model for spatial structure. The stencils are chosen to start at $k=20$. The dotted lines are at $\beta=-3 / 4,-2 / 3$. Bottom: Spectrally fitting $h$ from the thin jet model for spatial structure. Again, the stencils are chosen to start at $k=20$. The dotted lines are at $\beta=3 / 4,1$.

As discussed in Section 4, given a self-similar ansatz the local model (36-37) does not select exponents for the temporal behavior of extrema:

$$
h_{\min }(t) \sim\left(t_{c}-t\right)^{a} \quad \gamma_{\min }(t) \sim\left(t_{c}-t\right)^{\frac{a}{4}-\frac{1}{2}} \quad h_{x x \max }(t) \sim\left(t_{c}-t\right)^{\frac{a}{2}-1}
$$

If $a=2 / 3$, these exponents are those predicted by the assumption of self-similarity in solutions of the thin-jet model (29). To see if the local model selects any temporal exponents, we did a well-resolved $(n=8192)$ run. Surprisingly, we find that the temporal exponents are very close to those of the thin-jet solution, as is the spatial structure as revealed by its Fourier transform.

We first discuss the temporal behavior of the local model. Figure 16 shows fits to the leading-order powerlaw behavior of $\gamma_{\max }(t), h_{x x \max }(t)$, and $h_{\min }(t)$, and is to be compared with Figure 9. The error bars in the figures were computed by assuming an error of \pm .01 for the fitting of $h_{\min }(t)$ and finding that the exponents in (53) would suggest an error of \pm .0025 for fitting $\gamma$ and \pm .005 for fitting $h_{x x}$. The six figures suggest temporal exponents:

\begin{tabular}{ccc} 
extremum & Thin Jet Model & Local Model \\
\hline$h_{\min }(t)$ & 0.8 & 0.74 \\
$\gamma_{\max }(t)$ & -0.32 & -0.32 \\
$h_{x x \max }(t)$ & -0.66 & -0.63
\end{tabular}

The temporal exponents for the local model are in good agreement with the self-similarity exponents:

$$
h_{\min }(t) \sim\left(t_{c}-t\right)^{0.74} \quad \gamma_{\min }(t) \sim\left(t_{c}-t\right)^{-0.32} \quad h_{x x \max }(t) \sim\left(t_{c}-t\right)^{-0.63} .
$$



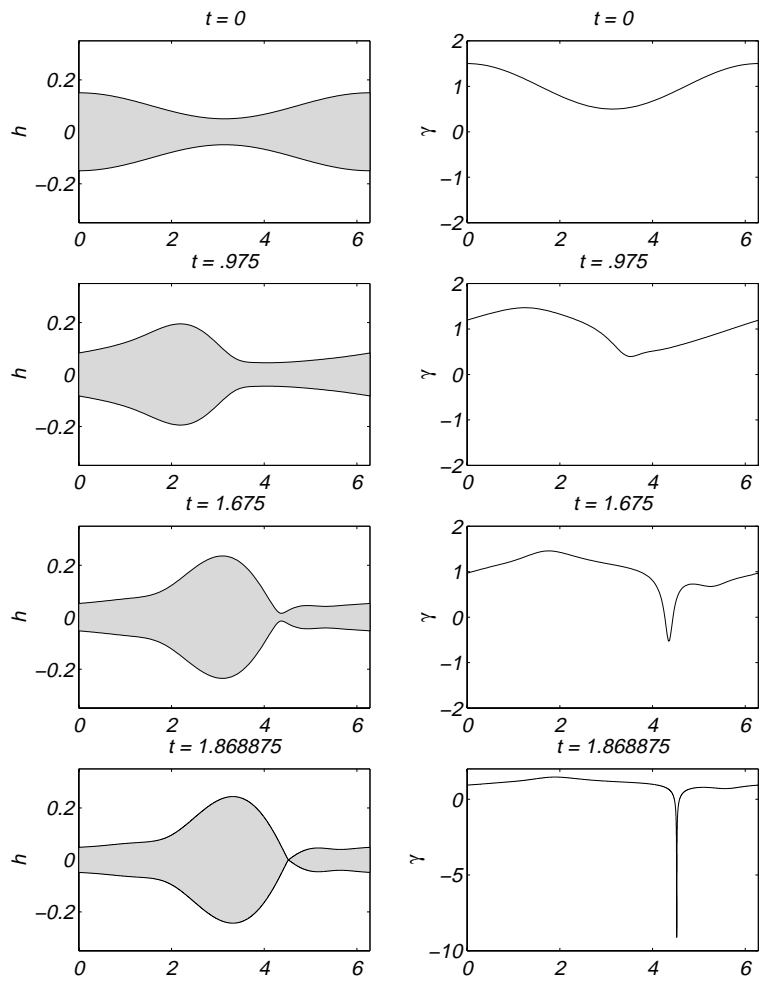

Figure 14. The long-time evolution of the local model.

We certainly cannot discount the possibility that a closer approach to the singularity time, through higher spatial and temporal resolution for both models, could lead to different exponents.

Figure 17 shows the fits of $\beta_{700}(k)$, the algebraic degree of complex singularities, from both the $\gamma$ and $h$ spectra near the pinching singularity time, and are the analogues of the bottom plot of Figure 11 and the bottom plot of Figure 12. In both the local model and the thin jet model, the spectra of $\gamma$ strongly suggest an algebraic degree of $\beta=-2 / 3$ for the complex singularity of $\gamma$. And in both models, the spectra of $h$ suggest a time-dependent algebraic degree for the complex singularity of $h$. The similarity between the figures is yet stronger in comparing Figure 17 to figures from fitting the (lower resolution) $n=8192$ simulation of the thin jet model. Specifically, the figures from fitting the $n=8192$ solutions of the thin jet simulation do not have as wide a "dip" in the low wave numbers in $\gamma$ and the values of $\beta$ for $h$ are not as close to $\beta=1$.

\subsection{Behavior of the thin jet model as the Weber number is varied.}

In the above, we studied the finite-time singularity for a small Weber number. A natural question is what happens to the singularity as the Weber number is taken to infinity. We performed a number of simulations with various Weber numbers using the initial data (48). As the Weber number increases, more modes become linearly unstable. All the simulations become singular in finite time: Figure 18 shows that as $W e$ increases to infinity, $t_{c}(W e)$ decreases to $t_{c}(\infty)$. We did not resolve these simulations well enough to determine the exact nature of the singularities. There are three clear options: $h$ touches down in finite time, $h$ shocks in finite time, and $h_{x x}$ blows up in finite time. The $W e<\infty$ simulations all appear to have $h$ touching down in finite time, although we cannot preclude that there might be a shock shortly before a touch-down.

Figure 19 shows the infinite Weber number simulation with initial data (48). It also has a finite-time singularity, but unlike the $W e<\infty$ simulations, $h$ remains well away from touching down. The evolution is 

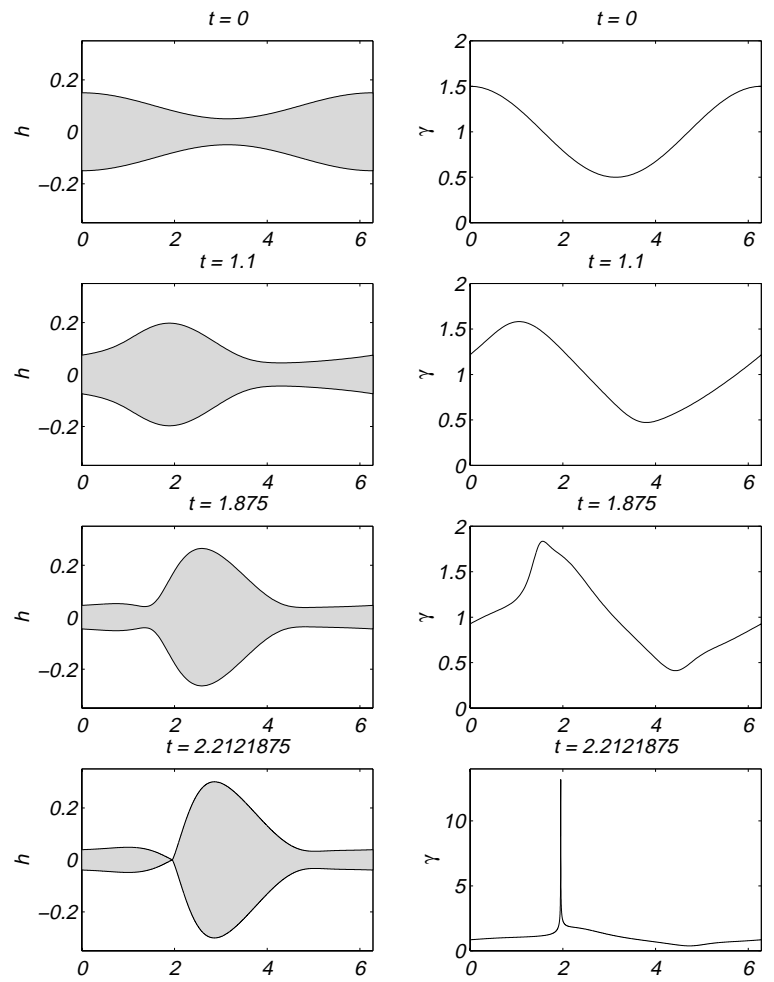

Figure 15. The long-time evolution of the thin jet model, given the initial data shown in Figure 14 .

initially similar: the extrema move to the right with the flow with the minimum of $h$ beginning to decrease. However, $h_{\min }$ has ripples in its wake and and one of these ripples shocks.

Figure 20 is the analogue of Figure 8 . The top plot of Figure 20 shows the positions of the different extrema - $h_{x \min }(t)$ and $\gamma_{\max }(t)$ occur at points that remain separate from one another, while $h_{x \max }(t)$ and $h_{x x \max }(t)$ occur at points that approach each other as the solution become singular. This is consistent with $h_{x} \uparrow \infty$, which would then force $h_{x x}$ to become infinite as well. The circle corresponds to the time when the $n / 2$ mode became active. In the bottom plot of Figure 20, we plot the singular times determined by three-point fits of the various extrema. This figure shows that $h_{x}$ and $h_{x x}$ become singular before $\gamma_{\max }$, $h_{\min }, \mathcal{K}_{\max }$, and $h_{x \min } \mathrm{do}^{3}$. Although $h_{x x}$ is blowing up, $\mathcal{K}$ (the curvature) is remaining bounded.

This behavior is at odds with simulations of a symmetric jet with no surface tension. Such a simulation is presented in HLS2, in which they find that the curvature blows up in finite time while the interface remains differentiable - the behavior of a single vortex sheet with no surface tension. Of course, our thin jet model assumes that $h_{x} \ll 1$, and hence the behavior of the infinite Weber number simulation is out of the regime of asymptotic validity.

\section{Conclusions}

In this paper, we derive and study models of a thin fluid jet that is separated from an outer immiscible fluid by interfaces under surface tension. We demonstrate numerically that both the thin jet model $(25-$ $26)$ and the local model $(36-37)$ have finite-time singularities. In the presence of surface tension $(W e<\infty)$,

\footnotetext{
${ }^{3}$ In this case, $h$ is not going to zero as $t \uparrow t_{c}$. However, $h_{\min }(t)$ is decreasing and fitting this decreasing function to $\left(t^{*}-t\right)^{p}$ determines some exponent $p$ and $t^{*}$ significantly larger than $t_{c}$.
} 

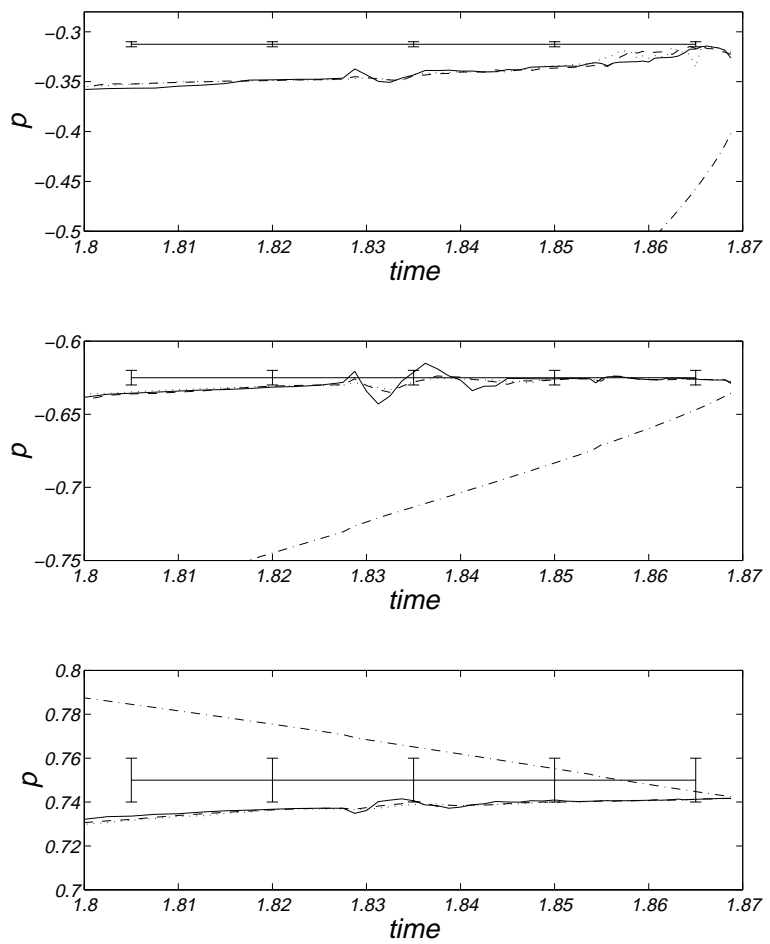

Figure 16. The evolution of the fitted temporal exponents for the local model. The dotdash line is the exponent found by three-point fittings. The remaining lines are from the fitting method described in Appendix B with weights $\alpha=.5, .75,1$. Top: Fitting $\min \{\gamma\}$ for the temporal exponent $p$. The line with error bars is $p=-.3125 \pm .0025$. Middle: Fitting $\max \left\{h_{x x}\right\}$ for the temporal exponent $p$. The line with error bars is $p=-.625 \pm .005$. Bottom: Fitting $\min \{h\}$ for the temporal exponent $p$. The line with error bars is $p=.75 \pm .01$.

the singularities are of a pinching type, where $h\left(x_{c}, t\right) \downarrow 0$ as $t \uparrow t_{c}$. In the absence of surface tension, the singularities are shocks where $\left|h_{x}\right| \uparrow \infty$. We study these singularities to determine their structure.

A natural expectation is that as the solution becomes singular it behaves in a self-similar fashion. The thin jet model determines all the temporal exponents of a self-similar solution (29). If the computed solution were close to self-similar behavior, the self-similar scaling would be apparent in the temporal evolution of extrema. To study this, we fit extrema ( $\operatorname{such}$ as $\left.h_{\min }(t)\right)$ to power laws and find that the singularities are in only partial agreement with self-similarity: $\gamma$ and $h_{x x}$ behave in close accordance with self-similarity, but $h$ does not. The thin jet model contains Hilbert transforms. which can be computed rapidly on a uniform mesh using a discrete Fourier transform. Future, more detailed, studies should use adaptive spatial meshes to better resolve the singular structure. Rapid evaluation of the Hilbert transform would then require use of summation methods such as adaptive Fast Multipole [18]. And so, while in this study the simulations appear to be highly resolved, we certainly cannot exclude that greater resolution is needed and that the solutions will eventually become clearly self-similar.

To further study the structure of the singularity, we examine the Fourier spectra of the solutions. We find that $\gamma(x, t)$ has an analytic structure corresponding to a conjugate pair of complex singularities of the form $(x-z(t))^{-2 / 3}$. There is also some evidence for a change of type in the singularity, away from an algebraic degree of $-2 / 3$, as the singular time is approached. For $h$, the singularity structure also seems to be described by a conjugate pair of complex singularities, but their algebraic degree appears time-dependent, and is apparently moving towards the value associated with a corner singularity. 

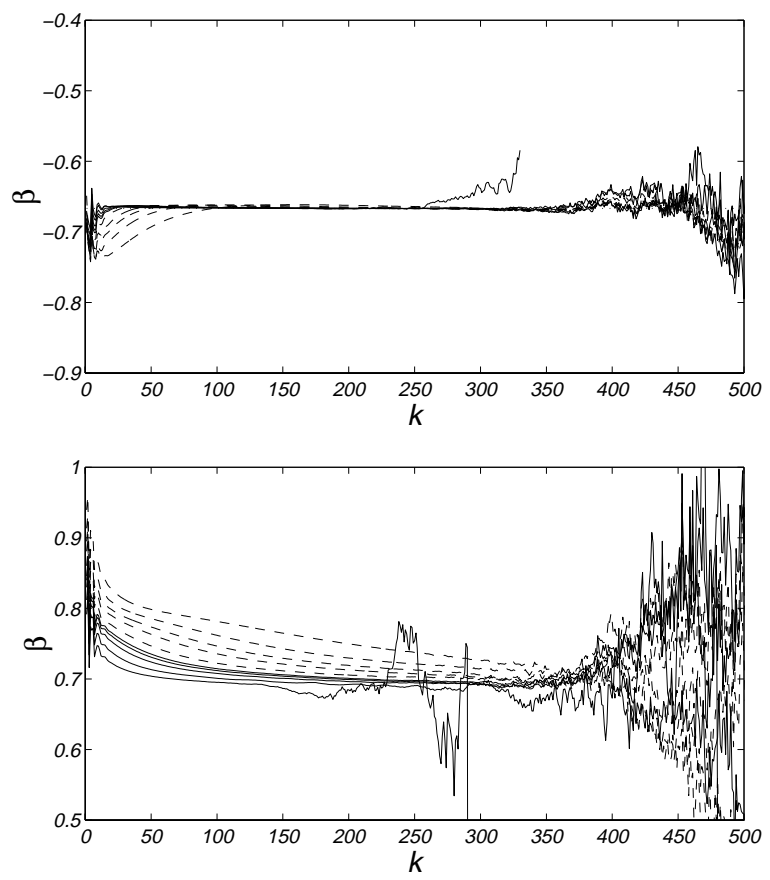

Figure 17. The solid lines correspond to times before the active part of the spectrum reaches the $n / 2$ mode, the dashed lines correspond to the times afterwards. Top: Using a stencil of length 700 to spectrally fit $\gamma$ from the local model for spatial structure. Bottom: Using a stencil of length 700 to spectrally fit $h$ from the local model for spatial structure.

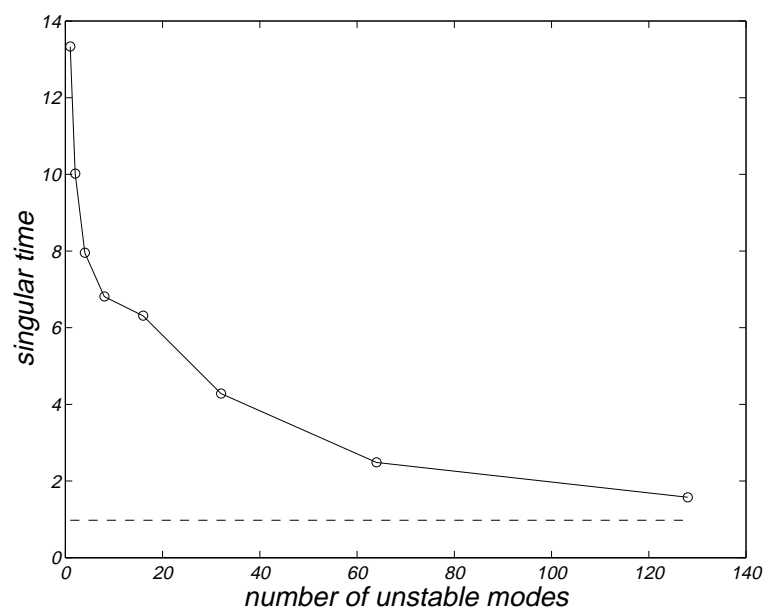

FigURE 18. The singular time $t_{c}$ as a function of the number of unstable modes.

The apparent formation of a corner singularity agrees with the numerical results of HLS2 in their study of the collapsing jet formed within the turns of the Kelvin-Helmholtz spiral. However, in their study they find strong evidence of self-similar temporal behavior in the width of the jet (here $h$ ), but a lack of strong evidence for such temporal scalings in $\gamma$ and the curvature $\mathcal{K}$. We, with Lowengrub, are currently studying the dynamics of a single symmetric fluid-fluid jet, models of which we study here. Our preliminary results 

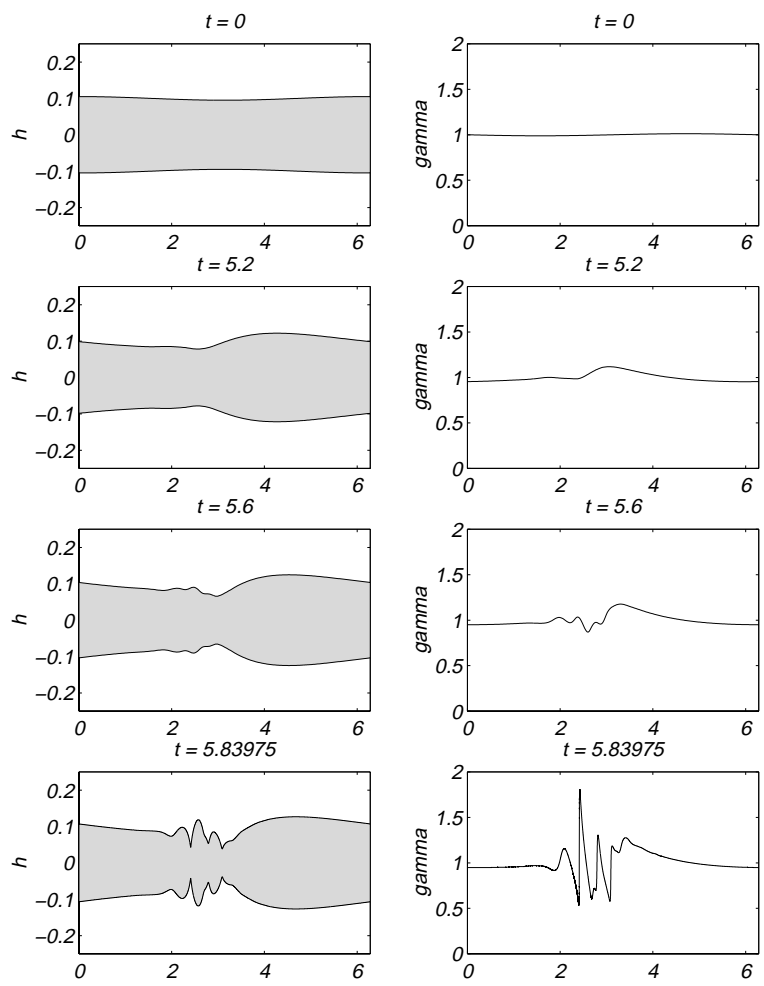

Figure 19. The evolution of the thin-jet model with zero surface tension $(W e=\infty)$. The initial data used in Figure 6 is used.

show a collapse of the jet width with temporal scalings that are not consistent with a self-similar collapse [22]. In fact, the temporal exponent, $a \sim .75-.76$, is quite close to that found in the simple model $(a \sim .74)$.

An analytic approach to studying singularity formation uses complex analytic methods. A real-valued analytic function, $h(x, t)$, is extended to the complex plane, as is its equation of evolution. If the complexified function has complex poles or zeros which reach the real axis in finite time, these can correspond to a finite-time singularity for the real-valued function. For a vortical flow, Moore's analysis provides a way of complexifying the flow and approximating non-local terms, such as Biot-Savart integrals, with local terms [10]. The approximate system is then studied to see if it has complex singularities which reach the real axis in finite time.

Siegel has performed a version of Moore's analysis for a single vortex sheet with surface tension (as studied in HLS2) and finds special solutions that form finite-time singularities [39]. In particular, he finds that before the singular time, both $\gamma$ and the sheet curvature have an analytic structure determined by conjugate pairs of complex singularities of the form $(x-z(t))^{-2 / 3}$. This agrees with our results for $\gamma$, though in Siegel's analysis $\gamma$ does not change type at the singular time. In Siegel's analysis, the curvature does change type, becoming a step function at the singularity time, giving a corner in the sheet profile. Siegel's analysis does not predict the formation of a pinching singularity - the corner singularity is isolated from other segments of the interface. This may be unsurprising, given that the Moore's approximation is local. While our models can be local, and yet give a pinching singularity, the shallow water form encodes the interaction of two opposing interfaces.

The temporal and spatial characteristics of pinching singularities found in our local model are almost identical to those in the thin jet model: the temporal exponents of the collapse are very close, and the spatial structure of the oncoming singularity (as revealed by the Fourier spectra) for both $h$ and $\gamma$ is very similar. Given its relative simplicity, we consider the local model of fundamental interest. 

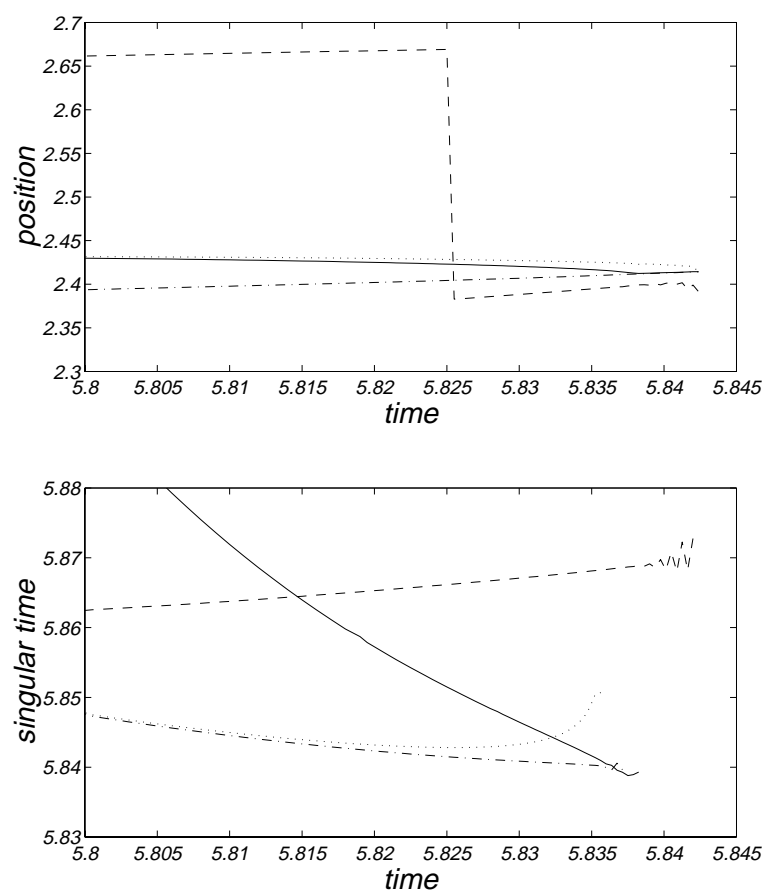

Figure 20. Top: The evolution of the position of extrema for the thin jet model with zero surface tension. The $x$-axis is time, the $y$-axis is the position of the extrema. The dotted line is the position of $\max \{\gamma\}$. The dashed line is the position of the $\min \left\{h_{x}\right\}$. The solid line is the position of $\max \left\{h_{x}\right\}$. The dot-dash line is the position of $\max \left\{h_{x x}\right\}$. Bottom: The evolution of the fitted singular time from three-point fittings for the thin jet model with zero surface tension. The $x$-axis is time, the $y$-axis is the fitted singular time. The solid line is the singular time from fitting $\max \left\{h_{x}\right\}$. The dashed line is the singular time from fitting $\min \{h\}$. The dot-dash line is the singular time from fitting $\max \left\{h_{x x}\right\}$. The dotted line is the singular time from fitting $\max \{\mathcal{K}\}$ (the curvature of the sheet).

Some similarity between the local and thin jet models is expected since the initial evolution is driven by the lower-order system (34-35) with the higher-order terms (the non-local terms and the dispersive term) entering late in the evolution. However, the idea that the pinching mechanism is related to "dispersing away the Burgers shock" is weakened since we find that further truncations of the local model continue to have finite-time pinching singularities. Specifically both

$$
h_{t}=-(h \gamma)_{x}, \quad \gamma_{t}=W e^{-1} h_{x x x}
$$

and

$$
h_{t}=-h \gamma_{x}, \quad \gamma_{t}=W e^{-1} h_{x x x}
$$

have finite-time pinching singularities but do not have a Burgers shock to disperse away. The second system keeps only the barest bones of a shallow water form. However, fitting the singularities of these two systems for their temporal and spatial behaviors, we find very different exponents from those seen in the thin jet and local models. Note also that neither system will produce a singularity in the $W e=\infty$ (zero surface tension) case.

Our studies of the thin jet and local models have produced further problems of interest. In the thin jet model, the local surface tension term appears to enslave the non-local terms. How it does this needs to be explained. For the local model, it is still unproven whether a uniform lower bound on $h, h \geq \alpha>0$, ensures 
regularity. Also, analysis may be brought to bear on proving the existence of finite-time singularities for the local model with positive initial $h$.

\section{Acknowledgments}

M.P. thanks B. Braams for long conversations about data-fitting and T. Sideris for the finite-time blow-up theorem for the local model. Both authors thank J. Lowengrub, D. Muraki, D. McLaughlin, and E. Tabak for useful discussions. M.P. was supported by an NSF post-doctoral fellowship while at Courant and by the Ambrose Monell Foundation while at the Institute for Advanced Study. M.J.S. acknowledges support from Department of Energy grant DE-FG02-88ER25053, National Science Foundation grants DMS-9396403 (PYI) and DMS-9404554, and the Exxon Educational Foundation.

\section{Appendix A. Reformulation of the Symmetric Jet Equations}

We first present the equations of motion for a single vortex sheet. While the normal component of the velocity is continuous across the interface, the tangential component of the velocity can be discontinuous, making the interface a vortex sheet. We require the interface to move with the fluid in the normal direction. The interface $\Gamma(t)$ is parametrized with a real variable $p$ and is denoted $\vec{X}(p, t)$, with unit normal vector $\vec{n}$ and unit tangent vector $\vec{s}$. The normal component of the velocity, $U(p)$ is given by

$$
\begin{gathered}
U(\vec{X}(p, t), t)=\vec{Q}(p, t) \cdot \hat{n} \quad \text { where } \\
\vec{Q}(p, t)=\frac{1}{2 \pi} P . V . \int_{\Gamma(t)} \gamma\left(p^{\prime}, t\right) \frac{\left(\vec{X}(p, t)-\vec{X}\left(p^{\prime}, t\right)\right)^{\perp}}{\left|\vec{X}(p, t)-\vec{X}\left(p^{\prime}, t\right)\right|^{2}} d p^{\prime}=\frac{1}{2}\left(\vec{u}_{1}(\vec{X}(p, t), t)+\vec{u}_{2}(\vec{X}(p, t), t)\right)
\end{gathered}
$$

The interface moves with the sum of this velocity (54) and $T \hat{s}$. The frame $T$ represents the additional freedom in the tangential direction arising from the freedom of parametrization of the vortex sheet:

$$
\vec{X}_{t}=\vec{Q}+T \hat{s} .
$$

The velocity jump in the tangential direction, $\tilde{\gamma}$, denoted the "true sheet strength", determines $\gamma(p, t)$, denoted the "unnormalized sheet strength":

$$
\frac{\gamma(p, t)}{\sigma_{p}(p, t)}=-[\vec{u}]_{\vec{X}(p, t)} \cdot \hat{s}=\tilde{\gamma}(\sigma, t)
$$

where $\sigma$ is the arclength.

Euler's equation (6) and the Young-Laplace condition (8) yield an evolution equation for the true sheet strength,

$$
\tilde{\gamma}_{t}+\tilde{\gamma} \vec{Q}_{\sigma} \cdot \hat{s}-\tilde{\gamma}_{\sigma} T=W e^{-1} \mathcal{K}_{\sigma}
$$

where $\mathcal{K}$ is the curvature of the vortex sheet. The above equation has been non-dimensionalized using the periodicity length $\lambda$ and a reference speed $\gamma_{c}$. If the immiscible fluids have different densities, these effects would appear in the above evolution equation, see HLS2. The dimensionless quantity is the Weber number

$$
W e=\frac{\lambda \gamma_{c}^{2}}{\bar{\tau}}
$$

where $\bar{\tau}=2 \tau /\left(\rho_{1}+\rho_{2}\right)$ is a rescaled surface tension. The Weber number reflects the balance between the dispersive effects of surface tension and the Kelvin-Helmholtz instability.

Parametrizing the sheet with a parameter $p$ that is advected with the flow,

$$
\vec{X}_{t}(p, t)=\vec{Q}(\vec{X}(p, t), t)+T(p, t) \hat{s}(\vec{X}(p, t), t),
$$


yields an equation for the unnormalized sheet strength

$$
\gamma_{t}-\frac{\partial}{\partial p}\left(T \frac{\gamma}{\sigma_{p}}\right)=W e^{-1} \mathcal{K}_{p}
$$

This equation becomes $\gamma_{t}=0$ if $W e=\infty$ and $T=0$, demonstrating the time-independence of the unnormalized sheet strength for a vortex sheet without surface tension in Lagrangian coordinates. Of course, $\gamma$ is an unphysical quantity and the true sheet strength, $\tilde{\gamma}$, which is a physical quantity, is not conserved in this case.

We now consider the case of a jet. A jet is bounded above and below by vortex sheets. The velocity of a point on one of these sheets is

$$
\vec{X}_{t}=\vec{Q}+T \hat{s}
$$

$\vec{Q}=(\tilde{u}, \tilde{v})$ is the average of the limiting velocities from above and below the sheet and has a boundary integral representation analogous to (54). In this article, we consider a horizontal jet with a top-bottom symmetry and take an Eulerian parametrization for the vortex sheets bounding the jet. We view the vortex sheets as the graphs of a function $h: \vec{X}(x, t)=(x, \pm h(x, t))$. Since

$$
\vec{X}_{t}=\vec{Q}+T \hat{s}=\left(\tilde{u}+T x_{\sigma}, \tilde{v}+T y_{\sigma}\right)
$$

for an Eulerian parametrization, the frame $T$ must be chosen so that

$$
\tilde{u}+T x_{\sigma}=0 \Longleftrightarrow T=-\frac{\tilde{u}}{x_{\sigma}}=-\tilde{u} \sqrt{1+h_{x}^{2}} .
$$

This choice of frame in $(56-57)$ yields

$$
\begin{aligned}
h_{t} & =y_{t}=\tilde{v}-\tilde{u} \frac{y_{\sigma}}{x_{\sigma}}=\tilde{v}-\tilde{u} h_{x} \\
\gamma_{t} & =-(\tilde{u} \gamma)_{x}+W e^{-1} \mathcal{K}_{x} .
\end{aligned}
$$

We now present the Fourier series reformulation of the Boundary integral formulation of the flow. The velocity field $\vec{Q}(x, y)=(u(x, y), v(x, y))$ at a point $(x, y)$ away from the interface can be represented in the complex notation:

$$
\begin{aligned}
u(x, y, t)-i v(x, y, t)= & \frac{1}{2 \pi i} \int_{-\infty}^{\infty} \frac{\gamma\left(x^{\prime}, t\right)}{x+i y-\left(x^{\prime}+i h\left(x^{\prime}, t\right)\right)} d x^{\prime} \\
& -\frac{1}{2 \pi i} \int_{-\infty}^{\infty} \frac{\gamma\left(x^{\prime}, t\right)}{x+i y-\left(x^{\prime}-i h\left(x^{\prime}, t\right)\right)} d x^{\prime} .
\end{aligned}
$$

If both $\gamma$ and $h$ are $2 \pi$-periodic, the above kernel is explicitly resummed:

$$
\begin{aligned}
u(x, y, t)-i v(x, y, t) & =\frac{1}{4 \pi i} \int_{0}^{2 \pi} \gamma\left(x^{\prime}, t\right) \cot \left(\frac{x+i y-\left(x^{\prime}+i h\left(x^{\prime}, t\right)\right)}{2}\right) d x^{\prime} \\
& \quad-\frac{1}{4 \pi i} \int_{0}^{2 \pi} \gamma\left(x^{\prime}, t\right) \cot \left(\frac{x+i y-\left(x^{\prime}-i h\left(x^{\prime}, t\right)\right)}{2}\right) d x^{\prime} \\
& =I_{1}(x, y, t)-I_{2}(x, y, t)
\end{aligned}
$$

The incompressibility and irrotationality of the fluid imply that $\vec{Q}^{*}(z)$ is a complex analytic function away from the interface. This suggests expressing the velocity field as a sum of analytic functions:

$$
u(x+i y, t)-i v(x+i y, t)=\sum_{k=-\infty}^{\infty} \alpha_{k}(h, \gamma) e^{i k(x+i y)}=\sum_{k=-\infty}^{\infty} \alpha_{k}(h, \gamma) e^{-k y} e^{i k x}
$$


We find the velocity $u_{1}(x, y, t)-i v_{1}(x, y, t)$ for a point completely inside the jet, $\{0 \leq y<h(x) \mid \forall x \in[0,2 \pi]\}$ as follows:

$$
I_{1}(x, y, t)=\sum_{k=-\infty}^{\infty} a_{k}(y, t) e^{i k x}
$$

where

$$
\begin{aligned}
a_{k}(y, t) & =\frac{1}{2 \pi} \int_{0}^{2 \pi} I_{1}(x, y, t) e^{-i k x} d x \\
& =\frac{1}{2 \pi} \int_{0}^{2 \pi} \frac{1}{4 \pi i} \int_{0}^{2 \pi} \gamma\left(x^{\prime}, t\right) \cot \left(\frac{x+i y-\left(x^{\prime}+i h\left(x^{\prime}\right)\right)}{2}\right) d x^{\prime} e^{-i k x} d x \\
& =\frac{1}{2 \pi} \int_{0}^{2 \pi} \gamma\left(x^{\prime}, t\right) \frac{1}{4 \pi i} \int_{0}^{2 \pi} \cot \left(\frac{x+i y-\left(x^{\prime}+i h\left(x^{\prime}\right)\right)}{2}\right) e^{-i k x} d x d x^{\prime} \\
& =\frac{1-\operatorname{sgn}(k)}{2} \frac{1}{2 \pi} \int_{0}^{2 \pi} \gamma\left(x^{\prime}, t\right) e^{k\left(h\left(x^{\prime}, t\right)-y\right)} e^{-i k x^{\prime}} d x^{\prime} \\
& =\frac{1-\operatorname{sgn}(k)}{2} e^{-k y} \frac{1}{2 \pi} \int_{0}^{2 \pi} \gamma\left(x^{\prime}, t\right) e^{k h\left(x^{\prime}, t\right)} e^{-i k x^{\prime}} d x^{\prime} .
\end{aligned}
$$

To evaluate the integral with respect to $d x$ in (62), we use the fact that the pole, $x^{\prime}+i\left(h\left(x^{\prime}, t\right)-y\right)$, is in the upper half plane for all $x^{\prime}$ and hence the residue appears only for $k<0$.

Similarly,

$$
I_{2}(x, y, t)=\sum_{k=-\infty}^{\infty} b_{k}(y, t) e^{i k x}
$$

where

$$
\begin{aligned}
b_{k}(y, t) & =\frac{1}{2 \pi} \int_{0}^{2 \pi} \gamma\left(x^{\prime}, t\right) \frac{1}{4 \pi i} \int_{0}^{2 \pi} \cot \left(\frac{x+i y-\left(x^{\prime}-i h\left(x^{\prime}\right)\right)}{2}\right) e^{-i k x} d x d x^{\prime} \\
& =-\frac{1+\operatorname{sgn}(k)}{2} e^{-k y} \frac{1}{2 \pi} \int_{0}^{2 \pi} \gamma\left(x^{\prime}, t\right) e^{-k h\left(x^{\prime}, t\right)} e^{-i k x^{\prime}} d x^{\prime}
\end{aligned}
$$

Here, there was no constraint that $x+i y$ be completely below the interface as the pole is in the lower half plane as long as $(x, y)$ is inside the jet.

Hence for all $x+i y$ completely inside the jet

$$
\begin{aligned}
u_{1}(x, y, t)-i v_{1}(x, y, t) & =\sum_{k=-\infty}^{\infty}\left(a_{k}(y, t)-b_{k}(y, t)\right) e^{i k x} \\
& =\sum_{k=-\infty}^{\infty} e^{-k y} \frac{1}{2 \pi} \int_{0}^{2 \pi} \gamma\left(x^{\prime}, t\right) e^{-|k| h\left(x^{\prime}, t\right)} e^{-i k x^{\prime}} d x^{\prime} e^{i k x} .
\end{aligned}
$$

This yields

$$
u_{1}(x, y, t)=\sum_{k=-\infty}^{\infty} \cosh (k y)\left(\frac{1}{2 \pi} \int_{0}^{2 \pi} \gamma\left(x^{\prime}, t\right) e^{-|k| h\left(x^{\prime}, t\right)} e^{-i k x^{\prime}} d x^{\prime}\right) e^{i k x}
$$

and

$$
v_{1}(x, y, t)=-i \sum_{k=-\infty}^{\infty} \sinh (k y)\left(\frac{1}{2 \pi} \int_{0}^{2 \pi} \gamma\left(x^{\prime}, t\right) e^{-|k| h\left(x^{\prime}, t\right)} e^{-i k x^{\prime}} d x^{\prime}\right) e^{i k x}
$$


We formally take $y \uparrow h(x, t)$ to integrate to find the flux,

$$
\begin{aligned}
\int_{0}^{h(x, t)} u_{1}(x, y, t) d y & =h(x, t) \frac{1}{2 \pi} \int_{0}^{2 \pi} \gamma\left(x^{\prime}, t\right) d x^{\prime} \\
+ & \sum_{k=-\infty, k \neq 0}^{\infty} \frac{1}{k} \sinh (k h(x, t))\left(\frac{1}{2 \pi} \int_{0}^{2 \pi} \gamma\left(x^{\prime}, t\right) e^{-|k| h\left(x^{\prime}, t\right)} e^{-i k x^{\prime}} d x^{\prime}\right) e^{i k x}
\end{aligned}
$$

The same calculation for a point $x+i y$ which is completely above the jet $\{y>h(x) \mid \forall x \in[0,2 \pi]\}$ yields

$$
u_{2}(x, y, t)-i v_{2}(x, y, t)=-\sum_{k=-\infty}^{\infty}(1+\operatorname{sgn}(k)) e^{-k y} \frac{1}{2 \pi} \int_{0}^{2 \pi} \gamma\left(x^{\prime}, t\right) \sinh \left(k h\left(x^{\prime}, t\right)\right) e^{-i k x^{\prime}} d x^{\prime} e^{i k x} \text {. }
$$

Taking the real and imaginary parts,

$$
u_{2}(x, y, t)=-\sum_{k=-\infty}^{\infty} \operatorname{sgn}(k) e^{-|k| y}\left(\frac{1}{2 \pi} \int_{0}^{2 \pi} \gamma\left(x^{\prime}, t\right) \sinh \left(k h\left(x^{\prime}, t\right)\right) e^{-i k x^{\prime}} d x^{\prime}\right) e^{i k x} .
$$

and

$$
v_{2}(x, y, t)=-i \sum_{k=-\infty}^{\infty} e^{-|k| y}\left(\frac{1}{2 \pi} \int_{0}^{2 \pi} \gamma\left(x^{\prime}, t\right) \sinh \left(k h\left(x^{\prime}, t\right)\right) e^{-i k x^{\prime}} d x^{\prime}\right) e^{i k x} .
$$

We formally take $y \uparrow h(x, t)$ to find $u_{1}(x, h(x, t), t)$ and $y \downarrow h(x, t)$ to find $u_{2}(x, h(x, t), t)$, yielding

$$
\begin{aligned}
\tilde{u}(x, t)=\frac{1}{2}\left(\sum_{k=-\infty}^{\infty} \cosh (k h(x, t)) \frac{1}{2 \pi} \int_{0}^{2 \pi} \gamma\left(x^{\prime}, t\right) e^{-|k| h\left(x^{\prime}, t\right)} e^{-i k x^{\prime}} d x^{\prime} e^{i k x}\right. \\
\left.\quad-\sum_{k=-\infty}^{\infty} \operatorname{sgn}(k) e^{-|k| h(x, t)} \frac{1}{2 \pi} \int_{0}^{2 \pi} \gamma\left(x^{\prime}, t\right) \sinh \left(k h\left(x^{\prime}, t\right)\right) e^{-i k x^{\prime}} d x^{\prime} e^{i k x}\right) .
\end{aligned}
$$

The system (58-59) with the above flux and $\tilde{u}$ has the eigenvalues (19) when linearized about $x(p, t)=p+\frac{\gamma}{2} t$, $h=\bar{h}, \gamma=\bar{\gamma}$. This shows that the above reformulation is linearly consistent with the boundary integral formulation (60). We discuss higher-order consistency in Appendix A.1.

In the case of a non-periodic jet, the above reformulation is similar, as $\cot (z)$ has the same simple poles as $1 / z$. The only difference is that sum with respect to $k$ is replaced with an integral with respect to $\xi$. Clearly, these reformulations can also be found for a single vortex sheet.

\section{A.1. Shallow water expansions.}

The above reformulation represents the velocity as

$$
\tilde{u}(x, t)=\sum_{k=-\infty}^{\infty} f_{k}(h(x, t), \gamma(x, t)) e^{i k x} .
$$

For a shallow water expansion of the velocity, we simply expand the Fourier coefficients $f_{k}(h, \gamma)=f_{k}(\epsilon H, \gamma)$ in powers of $\epsilon$. The reformulation allows us to expand in all orders at once. For example, recall equation (11): $h_{t}=-(\text { Flux })_{x}$. A Taylor series expansion of $e^{-\epsilon|k| H}=1-\epsilon|k| H+\ldots$ in the flux (65) yields

$$
\begin{aligned}
\operatorname{Flux}(\epsilon H)= & \sum_{l=1}^{\infty} \epsilon^{2 l} \sum_{n=0}^{l-1} \frac{(-1)^{l}}{(2 l-2 n-1) !(2 n+1) !} H(x, t)^{2 n+1} \mathcal{H}\left[\frac{d^{2 l-1}}{d x^{2 l-1}}\left(\gamma H^{2 l-2 n-1}\right)\right] \\
& +\sum_{l=1}^{\infty} \epsilon^{2 l-1} \sum_{n=0}^{l-1} \frac{(-1)^{l-1}}{(2 l-2 n-2) !(2 n+1) !} H(x, t)^{2 n+1} \frac{d^{2 l-2}}{d x^{2 l-2}}\left(\gamma H^{2 l-2 n-2}\right) .
\end{aligned}
$$


Rather than expanding the Fourier series reformulation, we could expand the boundary integral formulation. However, it is a messier expansion and while it can also be done to all orders in $\epsilon$, the source of the linear instability is obscured. At first glance, the two expansions have different forms; we verified that they are identical to the order needed for the thin jet model.

To study the validity of the asymptotic expansion, we assume the solutions are nearly self-similar with identical inner scales,

$$
h(x, t)=\epsilon\left(t_{c}-t\right)^{a} H\left(\frac{x-x_{c}(t)}{\left(t_{c}-t\right)^{b}}\right) \quad \gamma(x, t)=\left(t_{c}-t\right)^{c} \Gamma\left(\frac{x-x_{c}(t)}{\left(t_{c}-t\right)^{b}}\right) .
$$

We rescale time $\sigma s=\left(t_{c}-t\right)$ and space $x-x_{c}(t)=\sigma^{b} y$ where $s$ and $y$ are $O(1)$. With this rescaling, we find that the $\epsilon$ expansion of the evolution equation for $h, h_{t}=O(1)+O(\epsilon)+O\left(\epsilon^{2}\right)$, has terms

$$
O\left(\epsilon^{n}\right) \sim \epsilon^{n} \sigma^{c+n(a-b)} C_{n}(\eta)
$$

for $n \geq 0$. The functions $C_{n}(\eta)$ are determined by $H, H^{\prime}, \ldots H^{(n)}, G, G^{\prime}, \ldots G^{(n)}$, where the derivatives are with respect to $\eta=y / s^{b}$.

If $a<b$ then as $t \uparrow t_{c},\left|h_{x}\right| \uparrow \infty$, strongly violating the shallow water assumption. The case of $a=b$ also violates the shallow water assumption since it implies $h_{x}=O(1)$ as $t \uparrow t_{c}$. Given a fixed $\epsilon$ and $a \geq b$, then as $\sigma \downarrow 0$ the singular time approaches and (69) implies that an $O\left(\epsilon^{n}\right)$ term remains higher-order than an $O\left(\epsilon^{m}\right)$ term for all $m<n$.

For a fixed $\epsilon$, we are also interested in the range of times $\sigma$ for which the asymptotic series converges. In the following, we assume that

$$
\left|C_{n}(\eta)\right| \leq B_{n} \quad \forall \eta \in N
$$

where $N$ is the domain of the self-similar solution. We further assume that there is some $\epsilon_{0}$ and $\sigma_{0}$ for which

$$
\sum_{n=0}^{\infty}\left(\epsilon_{0} \sigma_{0}^{a-b}\right)^{n} B_{n}<\infty .
$$

Convergence for an interval of times $\sigma$ follows immediately:

$$
\sigma<\sigma_{0}\left(\frac{\epsilon_{0}}{\epsilon}\right)^{\frac{1}{a-b}} \Longrightarrow \sum_{n=0}^{\infty}\left(\epsilon \sigma^{a-b}\right)^{n} C_{n}(\eta)<\infty \quad \forall \eta \in N .
$$

If $a=b$ then, there is no constraint on $\sigma$ and the condition for uniform convergence of the asymptotic series is $\epsilon \leq \epsilon_{0}$. If $a>b$ then the smaller $\epsilon$ is, the larger the time period before the singular time $t_{c}$ on which the series converges.

On request, we will provide the details of both expansions based on the boundary integral formulation and Fourier reformulation.

\section{Appendix B. Fitting Extrema to Power Laws}

It is a common first guess to assume that near singularities the solution will have a self-similar behavior. That is, if the solution is going to zero at a point $x_{c}$ at a time $t_{c}$ then

$$
h(x, t)=\left(t_{c}-t\right)^{\alpha} H\left(\frac{x-x_{c}(t)}{\left(t_{c}-t\right)^{\gamma}}\right)+\text { higher-order corrections }
$$

where $x_{c}(t) \rightarrow x_{c}$ as $t \rightarrow t_{c}$. If the solution is exactly self-similar, then the minimum value of $h(x, t)$ will occur at $x=x_{c}(t)$ and $h_{\min }(t)$ is a pure power law:

$$
h_{\min }(t)=\left(t_{c}-t\right)^{\alpha} H(0) .
$$

In practice, the higher-order corrections to the self-similar ansatz may be non-negligible until times very close to the singular time. This presents two options. The first option is to compute to times as close to the singular time as possible and fit the data to a pure power law $a\left(t_{c}-t\right)^{p}$. This is an attractive option, but some 
problems may have computational aspects that make computing sufficiently close to the singular too costly. The second option is to fit not just the leading-order behavior, but some of the higher-order terms as well.

To fit the data to a pure power law, $a\left(t_{c}-t\right)^{p}$, one takes three sequential data points and uses Newton's method to solve the system of equations

$$
\begin{aligned}
y_{i-1} & =a\left(t_{c}-t_{i-1}\right)^{p} \\
y_{i} & =a\left(t_{c}-t_{i}\right)^{p} \\
y_{i+1} & =a\left(t_{c}-t_{i+1}\right)^{p} .
\end{aligned}
$$

This yields values for $a, p$, and $t_{c}$ for each data triplet. These quantities are then viewed as functions of time. For example, $\left\{t_{i}, t_{c}\left(t_{i}\right)\right\}$ can be studied to see if $t_{c}\left(t_{i}\right) \rightarrow T_{C}$ as $t_{i}$ goes to the end of the data available.

This procedure is ill-conditioned and its convergence depends strongly on the initial guess. For this reason, we go through the data backwards in time, taking the first data triplet to be the data closest to the singular time. The initial guess for the iteration can be usually found graphically or by using a slower minimization package, such as the one in matlab. The answers computed for the first triplet are then taken as the first guess for fitting the second triplet, and so on. This method frequently fails after a number of steps. The failure is usually due to not being able to invert the Jacobian needed for the Newton iteration.

While the above method is significantly better than making a guess at the singular time and plotting $\log \left(y_{i}\right)$ against $\log \left(t_{c \text { guess }}-t_{i}\right)$, the disadvantage of this approach is that it can yield unnecessarily gloomy results. Specifically, if one fits any data-sequence which doesn't have a pure power-law behavior and if the coefficient of the next-order term is large then the times $t_{i}$ will have to be very close to the singular time before the computed quantities $a\left(t_{i}\right), p\left(t_{i}\right)$, and $t_{c}\left(t_{i}\right)$ show any convincing "trend". This degree of resolution of a singularity can be difficult to obtain, especially in a code that has no local grid-refinement.

For this reason, we propose fitting the higher-order corrections, assuming that there is an analytic function $f$ such that

$$
y(t) \sim\left(t_{c}-t\right)^{p} f\left(\left(t_{c}-t\right)^{q}\right)=\left(t_{c}-t\right)^{b}\left(a_{0}+a_{1}\left(t_{c}-t\right)^{q}+a_{2}\left(t_{c}-t\right)^{2 q}+\ldots\right) .
$$

We then fit for $t_{c}, p, q$, and $a_{i}$. The three-point fitting described previously is ill-conditioned and fitting for higher-order terms is even more ill-conditioned. We find that the minimization problem can be made less ill-conditioned by fitting the analytic function $f$ with Chebyshev polynomials, rather than with a Taylor series:

$$
y(t) \sim\left(t_{c}-t\right)^{p} f\left(\left(t_{c}-t\right)^{q}\right)=\left(t_{c}-t\right)^{b}\left(a_{0} \phi_{0}\left(\left(t_{c}-t\right)^{q}\right)+a_{1} \phi_{1}\left(\left(t_{c}-t\right)^{q}\right)+\ldots\right) .
$$

We use the following method to compute the exponents, coefficients, and singular time.

- First estimate $a_{0}, p$, and $t_{c}$ by minimizing

$$
\sum_{i=1}^{3}\left(y_{i}-a_{0}\left(t_{c}-t_{i}\right)^{p}\right)^{2}
$$

The values of $p$ and $t_{c}$ from the three-point fit are used in weights in the rest of the fitting procedure. For this reason, we denote them by $p_{3}$ and $t_{c 3}$ in the following.

- A stencil length, $N$, is chosen and the values $a_{0}, p$, and $t_{c}$ from the previous step are used as a first guess to estimate $a_{0}, p, t_{c}, q$, and $a_{1}$ by minimizing

$$
\frac{1}{N} \sum_{i=1}^{N} w\left(t_{i}\right)^{2}\left(y_{i}-\left(t_{c}-t_{i}\right)^{p}\left(a_{0}+a_{1} \phi_{1}\left(\left(t_{c}-t\right)^{q}\right)\right)\right)^{2} .
$$

The weights are

$$
w\left(t_{i}\right)=\frac{1}{\left(t_{c 3}-t_{i}\right)^{\alpha p_{3}}} \quad \text { where } \quad \alpha \in[0,1] .
$$


The effect of the weights depends on the chosen value of $\alpha$. If $\alpha=0$, the weights are irrelevant. If $\alpha=1$, the weights would make the all terms in the sum of order 1 , if the values of $t_{c 3}$ and $p_{3}$ were the correct values. We discuss how to choose the exponent $\alpha$ later.

- The values $a_{0}, p, t_{c}, q$ and $a_{1}$ from the previous step are used as a first guess to estimate $a_{0}, p, t_{c}, q$, $a_{1}$, and $a_{2}$ by minimizing

$$
\frac{1}{N} \sum_{i=1}^{N} w\left(t_{i}\right)^{2}\left(y_{i}-\left(t_{c}-t_{i}\right)^{p}\left(a_{0}+a_{1} \phi_{1}\left(\left(t_{c}-t\right)^{q}\right)+a_{2} \phi_{2}\left(\left(t_{c}-t\right)^{q}\right)\right)\right)^{2} .
$$

- This can be continued as far as one chooses, minimizing

$$
\frac{1}{N} \sum_{i=1}^{N} w\left(t_{i}\right)^{2}\left(y_{i}-\left(t_{c}-t_{i}\right)^{p}\left(a_{0}+\sum_{j=1}^{n} a_{j} \phi_{j}\left(\left(t_{c}-t_{i}\right)^{q}\right)\right)\right)^{2}
$$

We usually stop at $a_{2}$, fitting for six parameters in all. To choose the number of parameters to fit, we first choose a number of parameters and then fix the second exponent $q$. We then fit the remaining quantities, finding the minimum value of the objective function $\operatorname{err}(q)$. Doing this for a range of values of $q$ between .3 and 3, we plot $(q, \operatorname{err}(q))$. Plot A of Figure 21 shows the result of this fitting. The ideal figure should have a clear minimum. We find that the more parameters we try to fit, the "flatter" the minimum becomes, corresponding to no value of $q$ being clearly best. Also, the graph is no longer smooth. These observations are simply a graphical demonstration of the ill-conditioning of the problem.

As for the length of the stencil, in some cases it is sufficient to take the stencil no longer than the minimum number of points needed to solve for the unknowns $(N=n+4)$. In other cases, the stencil has to be taken longer to avoid ill-conditioning. The two key aspects are how close the final data point is to the singular time and the length of the time window, $t_{N}-t_{1}$. We discuss this later.

All of the minimizations were done using the nonlinear least-squares minimization package, E04UPF, from the NAG library, Mark 16. We found that sometimes this routine would exit too early and were unable to choose the program parameters to avoid this. For this reason, we multiplied the objective function by a large number (1.e+20) and then minimized it. The NAG routine then took enough iterations to perform well on test problems with known behavior.

- After the minimization has been done, we can calculate the least mean square error

$$
\frac{1}{N} \sum_{i=1}^{N}\left(y_{i}-\left(t_{c}-t_{i}\right)^{p}\left(a+\sum_{j=1}^{n} a_{j} \phi_{j}\left(\left(t_{c}-t_{i}\right)^{q}\right)\right)\right)^{2} .
$$

to measure a goodness of fit. It is not clear that this is the best measure since if $y_{i} \rightarrow 0$ as $t_{i} \rightarrow 0$, the least mean square error emphasizes the data closest to the singular time the least.

We present results from a test problem in which $\left\{t_{i}\right\}=\{.1, .2, \ldots, 19.9\}$ and $y(t)=4(20-t)^{-\frac{5}{2}}-(20-t)^{\frac{5}{2}}$. The three-point fit yields $a_{0}=3.9888, p=-2.5029$, and $t_{c}=20.0002$. While the numbers from the threepoint fit already look fairly good, this is because the higher-order term is significantly higher order. We give this example to show that our method can actually find that small higher-order contribution. Once this higher-order correction is taken into account, the fit for the lower-order terms then becomes more accurate.

We fit twelve points from $18.8,18.9, \ldots 19.9$ using $\alpha=.5$ in the weight. The minimum value of the objective function is $1.5 \mathrm{e}-25$, the least mean square error is $8.3 \mathrm{e}-23$. 

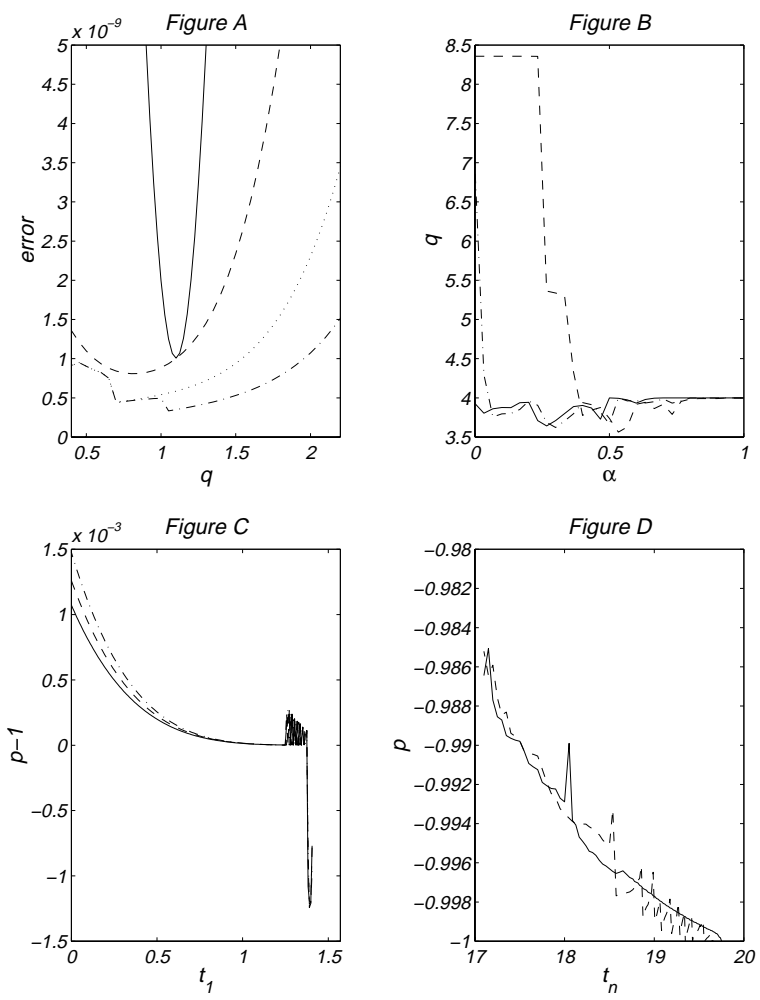

Figure 21. Figure A: The minimized objective function as a function of the higher-order temporal exponent $q$. The solid line corresponds to fitting for 5 parameters, the dashed line for 6 parameters, the dotted line for 7 parameters, and the dot-dashed line for 8 parameters. Figure B: The higher-order temporal exponent $q$ as a function of weight exponents $\alpha$. The dashed line corresponds to the shortest time window: $t_{1}=19.85, t_{N}=19.95$. The dot-dash line is for $t_{1}=19.835, t_{N}=19.95$. The solid line is for the longest time window: $t_{1}=19.82$, $t_{N}=19.95$. Figure $C$ : The leading-order temporal exponent $p$ as a function of time window length. The dot-dash line corresponds to weighting with exponent $\alpha=.5$. The dashed line to weighting with exponent $\alpha=.75$. The solid line to weighting with exponent $\alpha=1.0$. Figure D: The leading-order temporal exponent $p$ as a function of time $t_{N}$. The solid line corresponds to using Chebyshev polynomials for the fitting of higher-order terms. The dashed line corresponds to using powers for the fitting of higher-order terms.

\begin{tabular}{ccc} 
Notation & Exact Value & Computed Value \\
\hline$t_{c}$ & 20.0 & 20.000000000000 \\
$p$ & -2.5 & -2.4999999999998 \\
$a$ & 4.0 & 4.0000000000003 \\
$q$ & 5.0 & 4.9999999999979 \\
$a_{1}$ & -1.0 & -1.0000000000006 \\
$a_{2}$ & 0.0 & 0.0
\end{tabular}

Having more data points in the interval $[18.8,19.9]$ yields marginal improvement in these essentially exact quantities.

We now examine the dependence of the fit on the exponent $\alpha$ in the weights. We fit $y(t)=(20-t)^{-2.5}+$ $3(20-t)^{1.5}$ where $\left\{t_{i}\right\}=\{19,19.005, \ldots 19.995\}$. All fits are taken on a stencil that ends at the final time 
19.995. Plot B of Figure 21 shows fittings over three time windows. For each time window, we fix the exponent $\alpha$ in the weight, we minimize to fit the data, and we plot $(\alpha, q(\alpha))$. The exact answer for $q$ is 4 . The figure shows that the longer the time window, the more robust the fit. It also shows that for all time windows $\alpha=1$ is optimal. Finally, and most importantly, by looking at the values of $q$ for $\alpha=0$, we see that if no weights are used, the fitting is extremely poor.

We now examine the dependence of the fit on the length of the time window. We fit $y(t)=\cos (t)$. This has a singular time of $t_{c}=\pi / 2$, with exponent $p=1$. Plot $\mathrm{C}$ of Figure 21 shows fittings over all time windows for three different weights $\alpha=.5, .75,1.0$. We fix the point closest to the singular time $t_{N}=1.4451$ and fit over longer and longer intervals, taking $t_{1}$ to zero. We plot $\left(t_{1}, p-1\right)$ to see how the error depends on the length of the interval. The figure demonstrates that if $t_{1}$ is too close to $t_{N}$ then the error oscillates due to ill-conditioning. However, if $t_{1}$ is too far from $t_{N}$, the error grows because $\cos (t)$ is not a finite low-order power series. This type of figure allows us to choose a preferred length for the time window for further fitting studies.

A second test of the data-fitter is to apply it to data generated from numerically computing the solution to a PDE. To do this, we consider the local model (36-37)

$$
\begin{aligned}
h_{t}+\gamma h_{x} & =-h \gamma_{x} \\
\gamma_{t}+\gamma \gamma_{x} & =0 .
\end{aligned}
$$

As discussed in Section 4, this system has finite-time singularities, of the form

We choose initial data

$$
\gamma_{x \min }(t) \sim \frac{-1}{t_{c}-t} \quad h_{\max }(t) \sim \frac{1}{t_{c}-t} .
$$

$$
h_{0}(x)=\gamma_{0}(x)=0.1+0.05 \cos (x) .
$$

This initial data has been chosen so that the minimum of $\gamma_{0_{x}}$ and the maximum of $h_{0}$ occur at different points in space. For this initial data,

$$
\gamma_{x \min }(t)=\gamma_{x}(0, t)=-\frac{1}{20-t}
$$

The system is solved with a pseudo-spectral code, using Adams-Bashforth time-stepping. The times for point-doubling are chosen by looking at the spectrum of the solution and stopping the run before the active modes reach the $n / 2$ mode, to reduce the aliasing error. The $n=8192$ run is stopped when the conserved quantities $\int h$ and $\int \gamma$ are no longer conserved. A pseudo-spectral code is not the optimal approach to resolving singularities in the above system. A code with mesh-refinement would be better able to resolve the singularity. Since we ultimately apply this data-fitting scheme to data from a code with uniform mesh, we test it against data from such codes.

The data has the following time structure:

\begin{tabular}{ccl}
$\mathrm{n}$ & initial time & ending time \\
\hline 256 & 0.0 & 12.75 \\
1024 & 12.75 & 16.95 \\
2048 & 16.95 & 18.05 \\
4096 & 18.05 & 18.7625 \\
8192 & 18.7625 & 19.625
\end{tabular}

Fitting $\gamma_{x \min }(t)$ yields an extremely clean fit since it is an exact power law:

$$
\gamma_{x \min }(t) \sim(20.0000000-t)^{-1.0000000}\left(-1.0000000+4.4 e-11(20.0000000-t)^{0.5000000}+\ldots\right)
$$

Since the method also fits for higher-order corrections, it finds an exponent $q=1 / 2$ and zero for the higherorder coefficients. The reason it found this value of $q$ is simply that this was the first guess made for $q$.

Fitting $h_{\max }(t)$ is significantly harder. For initial data chosen so that the minimum of $\gamma_{0_{x}}$ and the maximum of $h_{0}$ occur at the same point, the extrema travel on the same characteristic, making $h_{\max }(t)$ an 
exact power law, and fitting $h_{\min }(t)$ with sequential data-triples would work extremely well. However, we do not study the data-fitting method on such initial data since for a general nonlinear problem we cannot expect to choose the initial data so fortunately.

We consider two issues in the following study of fitting $h_{\max }(t)$.

First, we take the the data up to the time $t_{N}=19.535$ and use different lengths of stencils to see the effect of different "time windows". We present the exponent $p$ and singular time $t_{c}$. The time $t_{N}$ is past the time where aliasing error comes into play, but is before the time when the solution becomes clearly inaccurate.

\begin{tabular}{llcc} 
initial time & time window & $t_{c}$ & $p$ \\
\hline 19.235 & 0.300 & 20.001478 & -1.010492 \\
19.025 & 0.600 & 20.000331 & -1.001909 \\
18.725 & 0.900 & 20.000087 & -1.000226 \\
18.425 & 1.200 & 20.000053 & -1.000159 \\
18.000 & 1.625 & 20.000022 & -1.000087 \\
17.500 & 2.125 & 19.999981 & -0.999915 \\
17.000 & 2.625 & 19.999934 & -0.999718 \\
16.150 & 3.475 & 19.999837 & -0.999312 \\
15.150 & 4.475 & 19.999695 & -0.998800 \\
14.150 & 5.475 & 19.999488 & -0.998155 \\
13.150 & 6.475 & 19.999223 & -0.997420
\end{tabular}

These computations show that the singular time and leading exponent are robust in that they are still clearly 20 and -1 over a window of length 6.5 , as long as the window includes times near to the singular time.

We now consider time windows of fixed length, which end at different times, to study what would have happened had the code run for a shorter length of time. In the following, "new exponent" means the exponent as calculated using the procedure using the weights with $\alpha=1$. "Old exponent" means the exponent that comes from simply fitting the same collection of data points to power law $a\left(t_{c}-t\right)^{p}$.

\begin{tabular}{llll} 
ending time & time window & new exponent & old exponent \\
\hline 19.625 & 0.975 & -1.0000 & -0.9954 \\
19.456 & 0.994 & -0.9990 & -0.9924 \\
19.269 & 0.994 & -0.9985 & -0.9900 \\
19.081 & 0.994 & -0.9980 & -0.9869 \\
18.856 & 1.006 & -0.9972 & -0.9833 \\
18.7625 & 1.013 & -0.9968 & -0.9814 \\
18.275 & 0.975 & -0.9948 & -0.9741 \\
18.05 & 1.0 & -0.9933 & -0.9683 \\
17.85 & 1.1 & -0.9920 & -0.9652 \\
17.35 & 1.0 & -0.9894 & -0.9543 \\
16.95 & 1.0 & -0.9855 & -0.9472
\end{tabular}

The data-fitting shows that by the time 18.05, the exponent is fairly clearly -1 . This means that this method of data-fitting only needs the simulation up to 2048 mesh-points. This is to be contrasted to fitting the data to a power law, yielding exponents that are suggestive but still inconclusive at the end of the 4096 mesh-point run (18.7625), needing an 8192 mesh-point run to resolve the exponent.

We mentioned previously that we found that fitting $f\left(\left(t_{c}-t\right)^{q}\right)$ in $(70)$ with powers of $\left(t_{c}-t\right)$ was more ill-conditioned than fitting it with Chebyshev polynomials. Plot D of Figure 21 shows two fittings of $\left(t_{1}, p\left(t_{1}, t_{N}\right)\right)$. The extremely jagged graph was computed by fitting $f\left(\left(t_{c}-t\right)^{q}\right)$ with powers of $\left(t_{c}-t\right)^{q}$, the smoother graph was computed by fitting $f$ with Chebyshev polynomials.

On request, we will provide Fortran programs of the data-fitting routine. 


\section{Appendix C. Fitting Complex Singularities}

As described in Section 5.3, Laplace's formula describes the asymptotic behavior of the Fourier transform of a function with complex poles or zeros:

$$
f(x) \sim(x-i \rho)^{\beta} \longrightarrow|\hat{f}(k)| \sim C e^{-\rho|k|} \frac{1}{|k|^{\beta+1}} g(1 / k) \quad \text { for } k \gg 1 \text { and } \beta>-1
$$

where $g$ is an analytic function.

Given $n$ mesh-points, the discrete Fourier transform determines $n / 2$ amplitudes: $|\tilde{f}(0)|, \ldots|\tilde{f}(n / 2-1)|$. The discrete Fourier amplitudes are related to the true Fourier amplitudes by

$$
|\tilde{f}(k)|^{2}=\sum_{j=-\infty}^{\infty}\left|\hat{f}\left(k+j \frac{n}{2}\right)\right|^{2} \sim \sum_{j=-\infty}^{\infty} e^{-2 \rho\left(k+j \frac{n}{2}\right)} \frac{1}{\left(k+j \frac{n}{2}\right)^{2(\beta+1)}} .
$$

If the radius of convergence $\rho$ is large then

$$
e^{-2 \rho\left(k+j \frac{n}{2}\right)} \frac{1}{\left(k+j \frac{n}{2}\right)^{2(\beta+1)}}<10^{-18} \quad \forall|j| \geq 1
$$

and there is no aliasing error - the discrete Fourier amplitude equals the true Fourier amplitude up to machine precision. To test the effect of aliasing error on fitting complex singularities, we choose $\rho$ and $\beta$ and generate an sequence of discrete Fourier amplitudes

$$
a_{k}=\sum_{j=-10}^{10} e^{-2 \rho\left(k+j \frac{n}{2}\right)} \frac{1}{\left(k+j \frac{n}{2}\right)^{2(\beta+1)}} \quad \text { for } k=0 \ldots n / 2 .
$$

We then fit $\left\{a_{k}\right\}$ for $\rho$ and $\beta$.

Figure 22 shows that aliasing error has negligible effect on this data fitting. Choosing $n=256$ and $\beta=-1 / 2$, we take six values of $\rho: .4, .3, .2, .1, .05, .025$. In the top plot of Figure 22 we plot $\left(k, \log \left(a_{k}\right)\right)$ to show the power spectrum. As the Fourier amplitudes are generated a priori, rather than by applying the Fourier transform to a function, there is no noise at the level of round-off error. Only $\rho=.4$ yields a spectrum without aliasing error. The bottom plot of Figure 22 shows the results of fitting the spectra with a stencil of length 30. The exponent $\beta=-1 / 2$ is determined cleanly for all values of $\rho$, despite the presence of aliasing error.

We now apply the data-fitting to the inviscid Burgers equation,

$$
\gamma_{t}+\gamma \gamma_{x}=0
$$

This is a simple example where the complex singularity changes algebraic degree when it hits the real axis. Using the method of characteristics, one can show analytically that $\beta(t)=1 / 2$ for $t<t_{c}$ but $\beta\left(t_{c}\right)=1 / 3$.

In the top plot of Figure 23 we present the results of fitting $\gamma$ for the algebraic degree $\beta$ where $\gamma$ is obtained from a pseudo-spectral computation with 512 mesh-points. The initial data was chosen to shock at time $t=20$. At a fixed time, we fit the spectrum by minimizing (51) over a stencil of length 30 . We plot $\left(k, \beta_{30}(k)\right)$. The solid lines corresponds to times before $t_{256}=15.75$, when the $n / 2$ mode is hit, the dashed lines correspond to times after $t_{256}$. As the previous example shows that aliasing error should not affect the data-fitting, we present $t_{256}$ as a lower bound for the time when the computation loses accuracy.

The top plot of Figure 23 shows that $\beta_{30}$ is clearly $1 / 2$ for a significant time and that at late times $\beta_{30}$ drifts down to $\beta=1 / 3$. The dashed curve with values nearest to $\beta=1 / 3$ was computed at time $t=20$, the singular time. It is striking that that this change of type is so robust that we can fit for it at the singular time $^{4}$.

In the middle plot of Figure 23 we plot the radius of convergence $\left(t, \rho_{30}(i, t)\right)$ for $i=1,6,11,16$. The radius of convergence is decreasing to zero although the curve is not concave down.

\footnotetext{
${ }^{4}$ This is not an option for the solutions of the thin-jet model (25-26) since the conjugate gradient iteration failed to converge before the singular time.
} 

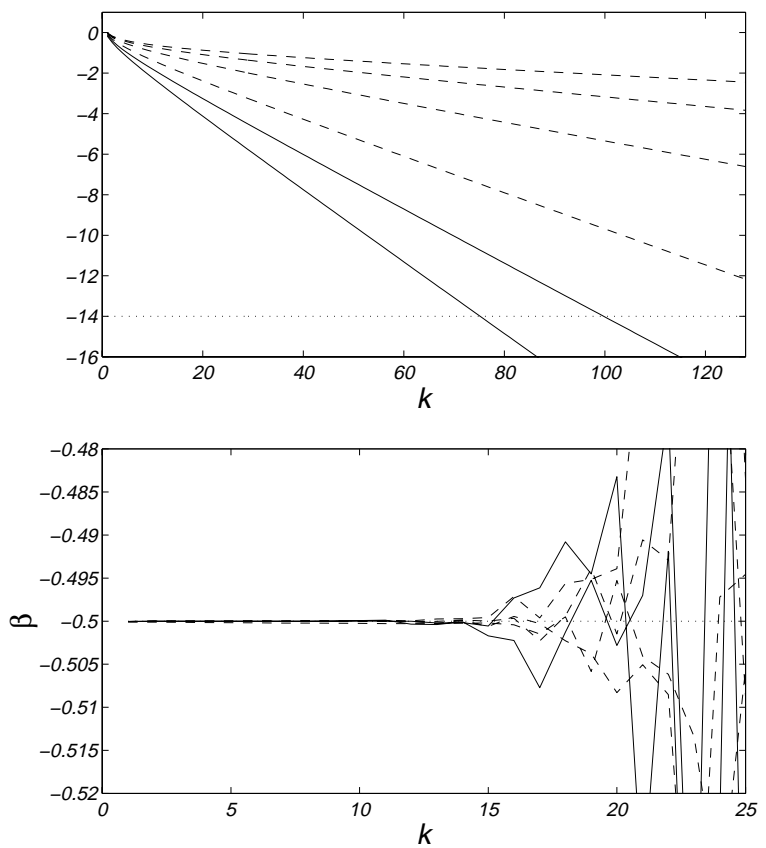

Figure 22. Top: The power spectrum for varying radii of convergence $\rho$. The dotted line corresponds to the spectrum of double precision round-off error. The solid lines are for $\rho=.4, .3$, whose active spectrum has not reached the $n / 2$ mode. The dashed lines have active spectrum past the $n / 2$ mode. Bottom: The results of fitting the power spectra shown in Figure 22 for the spatial exponent $\beta$. The true value is marked by a dotted line at $\beta=-1 / 2$.

As fitting for the algebraic degree will least ill-conditioned on stencils that include low wave-numbers, it is natural to try fitting over stencils of varying lengths. Specifically, we choose stencils that start at $k=1$ and stop at $L$. In the bottom plot of Figure 23 we plot $\left(L, \beta_{L}\right)$. The low-wave numbers in the stencil make the fitting problem less ill-conditioned - we can fit up to $\beta(200)$. The evidence for $\beta\left(t_{c}\right)=1 / 3$ is stronger in this figure.

Figure 23 is very similar to the the figure found when fitting

$$
\gamma(x, t)=\frac{\sin (x)}{\sqrt{\cos (x)+1+\rho(t)}} \quad \rho(t)=1-t .
$$

This function has square root poles $(\beta=-1 / 2)$ before the singular time and is a step function $(\beta=0)$ at the singular time.

In general, we found that the spectral data-fitting was quite ill-conditioned. Specifically, unless the data was in quadruple precision, taking a stencil of the same length as the number of unknowns yielded very little information. We refer the reader to D. Pugh's thesis [33] as well as to Sulem, Sulem, \& Frisch [40] for further information about fitting complex singularities.

\section{REFERENCES}

[1] R. Almgren, A. Bertozzi, and M. P. Brenner. Stable and unstable singularities in the unforced Hele-Shaw cell. Phys. Fluids, 8(6):1356-1370, 1996.

[2] G. R. Baker and A. Nachbin. Stable methods for vortex sheet motion in the presence of surface tension. to appear in SIAM J. Sci. Comp.

[3] G. R. Baker and M. J. Shelley. On the connection between thin vortex layers and vortex sheets. J. Fluid Mech., 215:161-194, 1990. 

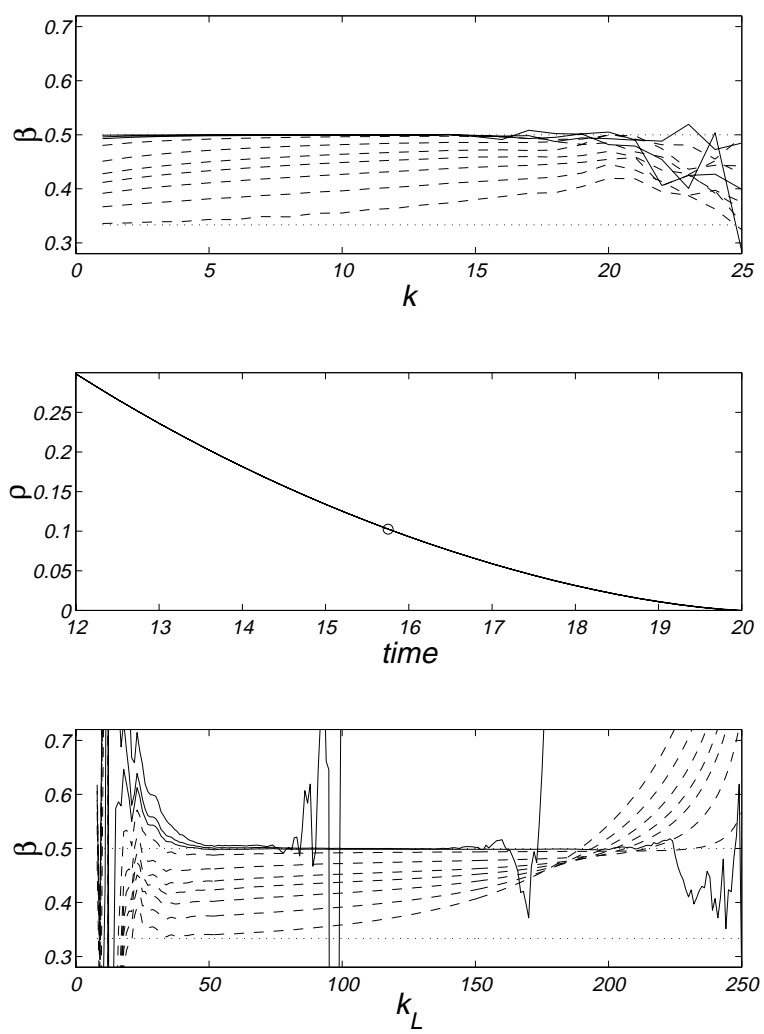

Figure 23. Top: Using a stencil of length 30 to spectrally fitting $\gamma$ from the inviscid Burgers equation for spatial structure. The solid lines are from fitting times before $t_{n / 2}$, the dashed lines from fitting times after $t_{n / 2}$. The dotted lines are at $\beta=1 / 2,1 / 3$. Middle: The evolution of the radius of convergence $\rho_{30}$ as a function of time. The circle denotes the time $t_{n / 2}$. Bottom: Using stencils of increasing length to spectrally fit $\gamma$ from the inviscid Burgers equation for spatial structure. The stencils start at $k=1$. The solid lines are from fitting times before $t_{n / 2}$, the dashed lines from fitting times after $t_{n / 2}$. The dotted lines are at $\beta=1 / 2,1 / 3$.

[4] J. T. Beale, T. Y. Hou, and J. S. Lowengrub. Growth rates for the linearized motion of fluid interfaces away from equilibrium. C.P.A.M., 47:1269-1301, 1993.

[5] S. E. Bechtel, M. G. Forest, and K. J. Lin. Closure to all orders in 1-d models for slender viscoelastic free jets: An integrated theory for axisymmetric, torsionless flows. Stability Appl. Anal. Contin. Media, 2(1):59-100, 1992.

[6] T. B. Benjamin, J. L. Bona, and J. J. Mahony. Model equations for long waves in nonlinear dispersive systems. Phil. Trans. Royal A, 272:47-78, 1972 .

[7] A. L. Bertozzi, M. P. Brenner, T. F. Dupont, and L. P. Kadanoff. Singularites and similarities in interface flows. In L. Sirovich, editor, Trends and Perspectives in Applied Mathematics, Applied Mathematical Sciences. Springer-Verlag, 1993.

[8] M. P. Brenner, J. R. Lister, and H. A. Stone. Pinching threads, singularities, and the number $0.0304 \ldots$ to appear in Phys of Fluids.

[9] R. E. Caflisch. Singularity formation for complex solutions of the 3d incompressible Euler equations. Physica D, 67:1-18, 1993.

[10] R. E. Caflisch, O. F. Orellana, and M. Siegel. A localized approximation method for vortical flows. SIAM J. Appl. Math., 50(6):1517-1532, 1990.

[11] Y.-J. Chen and P. H. Steen. Dynamics of inviscid capillary breakup: collapse and pinchoff of a film bridge. submitted to JFM, 1996. 
[12] P. Constantin, T. Dupont, R. Goldstein, L. Kadanoff, M. Shelley, and S. M. Zhou. Droplet breakup in a model of the Hele-Shaw cell. Phys. Rev. E, 47:4169-4181, 1993.

[13] J.-M. Delort. Existence de nappes de tourbillon en dimension deux. Journal of the A.M.S., 4(3):553-586, 1991.

[14] J. Eggers. Universal pinching of 3d axisymmetric free surface flow. Phys. Rev. Lett., 71:3458, 1993.

[15] J. Eggers and T. F. Dupont. Drop formation in a one-dimensional approximation of the Navier-Stokes equation. J. Fluid Mech., 262:205, 1994.

[16] R. Goldstein, A. Pesci, and M. J. Shelley. Topology transitions and singularities in viscous flows. Phys. Rev. Lett., 70:3043, 1993.

[17] R. Goldstein, A. Pesci, and M. J. Shelley. Attracting manifold for a viscous topology transition. Phys. Rev. Lett., 75:3665, 1995.

[18] L. Greengard and V. Rokhlin. A fast algorithm for particle summations. J. Comp. Phys., 73:325, 1987.

[19] T. Hou and H. Ceniceros. Convergence of a non-stiff boundary integral method for interfacial flows with surface tension. to appear in Math. Comp. December 1997.

[20] T. Hou, J. Lowengrub, and M. Shelley. Removing the stiffness from interfacial flows with surface tension. J. Comp. Phys, 114:312, 1994.

[21] T. Hou, J. Lowengrub, and M. Shelley. The long-time motion of vortex sheets with surface tension. Physics of Fluids, 9:1933-1954, 1997.

[22] M. Pugh J. Lowengrub and M. Shelley. The collapse of fluid-fluid jets with surface tension. In preparation, 1998.

[23] J. B. Keller and M. Miksis. Surface tension driven flows. SIAM J. App. Math., 43:268-277, 1983.

[24] R. Krasny. Desingularization of periodic vortex sheet roll-up. J. Comput. Phys, 65:292-313, 1986.

[25] R. Krasny. A study of singularity formation in a vortex sheet by the point vortex approximation. J. Fluid Mech., 167:65-39, 1986.

[26] E. A. Kuznetsov, M. D. Spector, and V. E. Zakharov. Formation of singularities on the free surface of an ideal fluid. Phys. Rev. E, 49(2):1283-1290, 1994.

[27] J. Lowengrub and M. Shelley. private communication, 1996.

[28] J. C. Luke. A variational principle for a fluid with a free interface. J. Fluid Mech., 27:395-397, 1967.

[29] D. McLaughlin. private communication, 1996.

[30] D. Meiron, G. R. Baker, and S. A. Orzag. Analytic structure of vortex sheet dynamics. part 1. Kelvin-Helmholtz instability. J. Fluid Mech., 114:283-298, 1982.

[31] D. W. Moore. The spontaneous appearance of a singularity in the shape of an evolving vortex sheet. Proc. R. Soc. London A, 365:105-119, 1979.

[32] D. T. Papageorgiou. Analytical description of the breakup of liquid jets. J. Fluid Mech., 301:109, 1995(a).

[33] D. A. Pugh. Development of vortex sheets in Boussinesq flows - formation of singularities. PhD thesis, Imperial College, London, 1989.

[34] Y. Saad and M.R. Schultz. GMRES: A generalized minimum residual method for solving nonsymmetric linear systems. SIAM J. Sci. Stat. Comput., 7:856, 1986.

[35] P. G. Saffman. Vortex dynamics. Cambridge Monographs on Mechanics and Applied Mathematics. Cambridge University Press, 1992.

[36] P. G. Saffmann and G. R. Baker. Vortex interactions. Ann. Rev. Fluid Mech., 11:95, 1979.

[37] M. J. Shelley. A study of singularity formation in vortex-sheet motion by a spectrally accurate vortex method. J. Fluid Mech., 244:493-526, 1992.

[38] T. Sideris. private communication, 1995.

[39] M. Siegel. A study of singularity formation in the Kelvin-Helmholtz instability with surface tension. SIAM J. Appl. Math., $55(4), 1995$.

[40] C. Sulem, P.-L. Sulem, and H. Frisch. Tracing complex singularities with spectral methods. J. Comp. Phys, 50:138-161, 1983.

[41] L. Ting and J. B. Keller. Slender jets and thin sheets with surface tension. SIAM J. Appl. Math., 50(6):1533, 1990.

[42] G. Tryggvason, W. J. A. Dahm, and K. Sbeih. Fine structure of vortex sheet rollup by viscous and inviscid simulation. J. Fluids Engin., 113:31, 1991.

[43] V. E. Zakharov. J. Appl. Mech. Tech. Phys., 2:190, 1968.

EMAIL: pugh@math.upenn.edu, shelley@cims.nyu.edu

Courant Institute, New York University, New York, NY 10012 